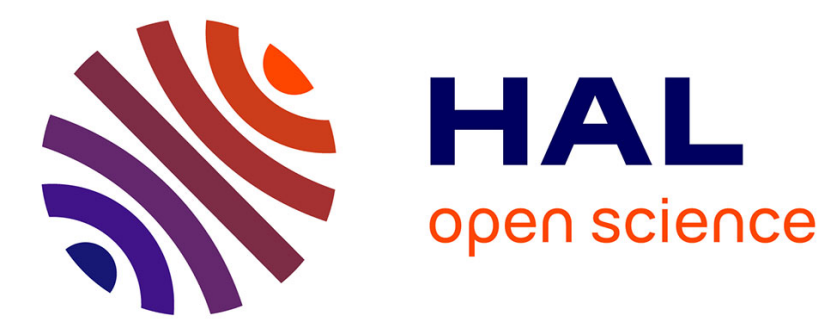

\title{
Initial Condensate Composition during Asteroid Impacts
}

D. de Niem, Ekkehard Kührt, U. Motschmann

\section{- To cite this version:}

D. de Niem, Ekkehard Kührt, U. Motschmann. Initial Condensate Composition during Asteroid Impacts. Icarus, 2009, 196 (2), pp.539. 10.1016/j.icarus.2008.03.012 . hal-00593402

\section{HAL Id: hal-00593402 \\ https://hal.science/hal-00593402}

Submitted on 15 May 2011

HAL is a multi-disciplinary open access archive for the deposit and dissemination of scientific research documents, whether they are published or not. The documents may come from teaching and research institutions in France or abroad, or from public or private research centers.
L'archive ouverte pluridisciplinaire HAL, est destinée au dépôt et à la diffusion de documents scientifiques de niveau recherche, publiés ou non, émanant des établissements d'enseignement et de recherche français ou étrangers, des laboratoires publics ou privés. 


\section{Accepted Manuscript}

Initial Condensate Composition during Asteroid Impacts

D. de Niem, E. Kührt, U. Motschmann

PII:

S0019-1035(08)00127-9

DOI: $\quad$ 10.1016/j.icarus.2008.03.012

Reference: $\quad$ YICAR 8638

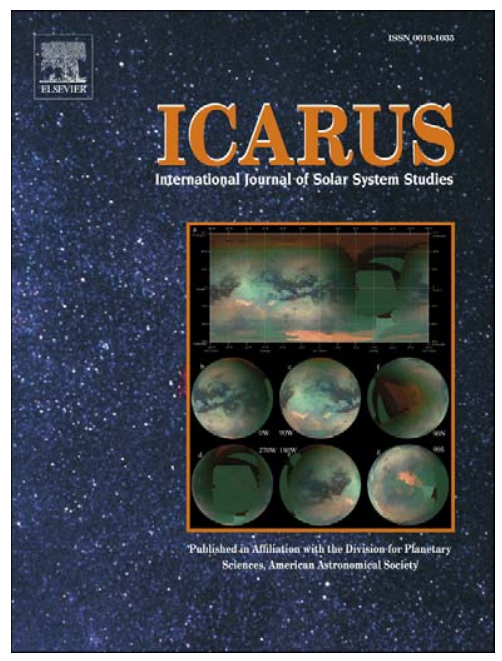

To appear in: Icarus

Received date: 11 May 2007

Revised date: 2 December 2007

Accepted date: 8 March 2008

Please cite this article as: D. de Niem, E. Kührt, U. Motschmann, Initial Condensate Composition during Asteroid Impacts, Icarus (2008), doi: 10.1016/j.icarus.2008.03.012

This is a PDF file of an unedited manuscript that has been accepted for publication. As a service to our customers we are providing this early version of the manuscript. The manuscript will undergo copyediting, typesetting, and review of the resulting proof before it is published in its final form. Please note that during the production process errors may be discovered which could affect the content, and all legal disclaimers that apply to the journal pertain. 


\title{
Initial Condensate Composition during Asteroid Impacts
}

\author{
D. de Niem ${ }^{a, *} \quad$ E. Kührt ${ }^{a} \quad$ U. Motschmann ${ }^{a, b}$
}

* Corresponding author E-Mail address: detlef.deniem@dlr.de

${ }^{a}$ DLR Institute of Planetary Research, Rutherford Str. 2, D-12489 Berlin, Germany

${ }^{b}$ Institute of Theoretical Physics, Technical University of Braunschweig, Mendelssohnstrasse 3, D-38106 Braunschweig, Germany

Pages: 43

Tables: 3

Figures: 8 
Running head:

Condensate Composition during Asteroid Impacts

Editorial correspondence to:

Dr. D. de Niem

Institut für Planetenforschung

Deutsches Zentrum für Luft- und Raumfahrt

Rutherford Str. 2

D-12489 Berlin

Germany

Phone: +493067055316

Fax: +493067055 340

E-mail address: detlef.deniem@dlr.de 


\begin{abstract}
A model has been developed for the chemical composition of early-time condensates forming during planetary impacts at velocities sufficient for complete evaporation of the impactor $\left(\sim 22 \mathrm{~km} \mathrm{~s}^{-1}\right)$. Hydrodynamics is approximated as hemispheric expansion of a chondritic impactor described with the help of a new semi-analytic equation of state. The gas phase is a mixture of a large number of molecular and mono-atomic species, whereas the Helmholtz free energy of the condensed phase is approximated as sum of a zero-temperature contribution and an Einstein-Debye solid for the thermal part. Interpolation of the free energy between the states of dense solids and molecular gases is used over the wide range of densities and pressures. Chemical equilibrium calculations, performed in a post-processing step, begin at conditions near the coexistence curve as obtained during hydrodynamic simulations, then the condensed phase contains 31 compound species, among them the most important oxides, sulphides, silicates as well as pure elements occurring in a liquid phase at sufficiently high temperature. The composition of the resulting liquid condensate in terms of major elements is compared to that of spinel inclusions in spherules found worldwide at the K-P boundary. Key words: Cratering, Earth, Impact Processes, Meteorites
\end{abstract}




\section{Introduction}

Impacts of meteorites, asteroids and comets on the earth are capable of causing vaporization of most of the impactor and a comparable or larger volume of target rocks (Pierazzo et al., 1997, 1998). Velocities in large terrestrial or lunar meteorite impacts are sufficient for the vaporisation of silicates; the average impact velocity of near earth objects (NEOs) is $\sim 20 \mathrm{~km} \mathrm{~s}^{-1}$ (Stuart and Binzel, 2004). In contrast, the average mutual collision velocities of main-belt asteroids of about $5 \mathrm{~km} \mathrm{~s}^{-1}$ (Bottke et al., 1994) are too low for significant evaporation. On the other hand, evaporation of the impactor can be expected during impact on the surfaces of the Galilean satellites, where the relative velocity is greatly enhanced due to the gravitational field of Jupiter (Zahnle et al., 1998). In the case of the Earth, the Cretaceous-Paleogene (KP) stratigraphic boundary is related to the impact of an extraterrestrial body (Alvarez et al., 1980) forming the Chicxulub crater, and some material has been interpreted as condensation product (Kyte and Bostwick, 1995). The K-P boundary is globally characterised by a layer containing spinel-bearing spherules, where the maximum iridium anomaly has been found (Smit, 1999; Montanari and Koeberl, 2000). Additionally, nanophase iron particles occur which also may have been formed as condensation product in the so-called fireball (Wdowiak et al., 2001). Nanophase dust may result due to a secondary peak in a multi-modal size distribution obtained in a kinetic model of condensation (de Niem, 2002), these calculations did not address the chem- 
ical composition. Space weathering on the lunar and asteroidal surfaces is thought to be partly caused by condensation of nanophase iron particles formed in a vapour in meteorite impacts (Keller and McKay, 1993; Hapke, 2001; Sasaki et al., 2002). On the Moon, velocities sufficient to cause significant evaporation of iron-bearing minerals can be reached during impact of meteors of cometary origin or for asteroidal impactors with large orbital eccentricities. Interestingly, meteor showers from 21P/Giacobini-Zinner show chondritic abundance ratios of $\mathrm{Na}: \mathrm{Fe}$ and $\mathrm{Mg}: \mathrm{Fe}$ in the spectra, and a temperature of ablation products of about $4000 \mathrm{~K}$ has been estimated, see Borovička et al. (2007). However, to infer the meteoride composition from such observations requires consideration of chemical fractionation, see Shaefer and Fegley (2004).

Considerable effort has been made to understand if spinels can form as condensation products from specific impacts, e.g. Chicxulub (Ebel and Grossman, 2005). According to Siret and Robin (2003) direct condensation of solid spinel crystals from the vapour phase is impossible. Earlier Robin et al. (1991) interpreted them as ablation residues during atmospheric entry, necessarily unrelated to the target rock composition. Kyte and Bostwick (1995) found that Ni-rich magnesiowüstite is preserved and intergrown with magnesioferrite spinel in the core of some spinel crystals. Because Ni-rich magnesiow üstite forms at temperatures even higher than $2300 \mathrm{~K}$, they suggested an origin in a hot fireball, where metal oxides dominate the liquid composition relative to silicates. Ebel and Grossman (2005) obtain favourable 
conditions for spinel formation in a silicate liquid in equilibrium with the gas phase at temperatures ranging from 1400 to $2400 \mathrm{~K}$. Their vapour plume model assumes complete mixing of the target and impactor components predicted by Pierazzo et al. $(1997,1998)$, prior to condensation. Calculations of chemical equilibrium by Ebel and Grossman (2005) have been confined to $T<2400 \mathrm{~K}$ and used a $(P, T)$ path extracted from hydrocode calculations. These hydrocode calculations (Pierazzo et al., 1997, 1998) used a different equation of state (EoS) in the gas phase than the chemical equilibrium solver. A hydrodynamic model of a condensing vapour plume requires an EoS not only in the high-pressure region, where data are available from shock wave experiments along the principal Hugoniot, mainly, but in a wide range of temperatures, pressures and densities. Shock wave experiments do not measure temperature and entropy directly, which are more important than pressure or particle velocity, in the context of condensation. Moreover, experiments corresponding to impact velocities in excess of $15 \mathrm{~km} \mathrm{~s}^{-1}$ are barely feasible with geomaterials. There are a number of laser experiments devoted to impact condensation and evaporation using meteoritic or analogue material, e.g. Moroz et al. (1996); Sasaki et al. (2002) however these experiments do not reproduce all thermodynamic conditions of a planetary impact: pressure, temperature, entropy, and do not lead to a full theoretical understanding of the observed compositions.

In this work, we investigate the simultaneous hydrodynamic, thermodynamic and chemical evolution of impact-created vapour numerically. In the 
hydrodynamic runs, thermodynamics is treated consistently up to the point where the coexistence curve of the vapour with the condensed phase is intersected. In Chapter 2 we describe our approach for the equation of state (EoS). Following Thompson and Lauson (1972); Bobrovskii et al. $(1974,1976)$ and Melosh (2007), we use an interpolation formula for the free energy to generate the EoS. Our equation of state is a semi-analytical development similar to ANEOS (Thompson and Lauson, 1972) with the additional property that it asymptotically approaches a mixture of ideal gases in chemical equilibrium at low densities. This mixture is constructed with some realism, including 26 mono-atomic and 183 molecular species, relevant in work about condensation in the early solar system (Sharp and Huebner, 1990).

In the third Chapter, using the derived EoS, we simulate the hydrodynamic expansion of an impact-generated vapour plume into a surrounding atmosphere and obtain liquid compositions in a post-processing step. The algorithm is a variant of our Eulerian multi-material volume-of-fluid method (de Niem et al., 2007), adapted to spherical symmetry. Back-reaction of condensation on the hydrodynamics of the expanding cloud is neglected. This is a valid approximation as long as the mass fraction of condensates remains small and the purpose of hydrodynamic simulation is to obtain the thermodynamical state variables at the onset of condensation only. The impactor is modelled as a carbonaceous chondritic object, using the elemental abundances compiled by Lodders (2003, her table 3 column 6) with modifications for elements like oxygen and hydrogen. We start with initial conditions for 
density, internal energy and pressure resulting from an impact with a given velocity, using the planar impact approximation (Melosh, 1989). Results are presented for two sets of initial conditions. A post-processing step, obtaining condensate compositions, is invoked when the boundary to the gas-liquid coexistence region is intersected in some computational cell, although the hydrodynamic run is continued until most of the material is affected.

In Chapter 4 we investigate the chemical equilibrium and condensate compositions and compare the overall elemental abundances of the refractive liquid to that of magnesioferrite spinels contained inside spherules from the global Cretaceous-Paleogene boundary layer analysed by Kyte and Bostwick (1995). For a model of the liquid phase as a single ideal solution, depending on initial conditions and composition, condensates begin to form at $T>4000$ K. The results and some approximations are critically discussed in Chapter 5.

\section{Analytical equation of state formulation in impact simulations}

\subsection{General thermodynamic considerations}

Following Thompson and Lauson (1972), our analytical equation of state (EoS) is based on the free energy per unit of mass $f$ as generating thermodynamic potential. In this way, a thermodynamically consistent EoS is 
developed. The free energy is the sum of three parts

$$
f=f_{c}+f_{E}+f_{\Psi}
$$

where $f_{c}$ is the zero-temperature ('cold') part. Thermal contributions of the condensed and gaseous phases (Zel'dovich and Raizer, 1967; Anderson, 1989) are denoted by $f_{E}$ and $f_{\Psi}$, respectively. The term $f_{E}$ is the free energy of an Einstein solid - a collection of 3 harmonic oscillators per atom - and $f_{\Psi}$ interpolates between the free energy of a mixture of gases and that of the condensed phase. The third term is essential for proper asymptotics in the case of evaporation. It is needed to connect high- and low-density states at super-critical temperatures and to construct the boundary of the twophase mixture region (Thompson and Lauson, 1972; Bobrovskii et al., 1974; Melosh, 2007). The interpolation term is such that it suppresses the lattice contributions of the condensed phase in the dilute gas limit, see section 2.3. In the region of liquid-vapor coexistence, a Maxwell construction has to be applied (Thompson and Lauson, 1972) if hydrodynamic calculations enter this part of thermodynamic space.

Previous models either did not address the full problem of chemical equilibrium with molecular species (Thompson and Lauson, 1972) in the gas phase, or are specialized to a gas phase with a single molecular species (Bobrovskii et al., 1974, 1976; Melosh, 2007), so chemical equilibrium considerations have been oversimplified. A recent application of the idea of Bobrovskii 
et al. (1976) by Ivanov and Deutsch (2002), investigated the decomposition of $\mathrm{CaCO}_{3}$ relating to the possible climate catastrophe following the Chicxulub impact.

\subsection{Zero-temperature part}

We use a simple model for the zero-temperature part of the free energy, $f_{c}$, distinguishing expansion $\left(\rho<\rho_{0}\right)$ and compression $\left(\rho \geq \rho_{0}\right)$ where $\rho_{0}$ is the density of the condensed phase at $T=0$ and $P=0$. Following Bobrovskii et al. $(1974,1976)$ or Melosh $(2007)$, for expansion $\left(\rho<\rho_{0}\right)$, we take a Lennard-Jones-like potential

$$
f_{c}=\frac{A}{\rho_{0}(\beta-\alpha)}\left(\frac{\eta^{\beta-1}-1}{\beta-1}-\frac{\eta^{\alpha-1}-1}{\alpha-1}\right)
$$

with $\eta:=\rho / \rho_{0}, A$ is the bulk modulus at $\rho=\rho_{0}, \alpha$ and $\beta$ are dimensionless parameters with $\alpha<\beta$ in order that a minimum results at $\rho=\rho_{0}$. Rather than giving these parameters directly, both $\rho_{0}$ and $A$ may be determined by matching the bulk modulus and density at normal conditions. The exponent $\beta$ is determined, knowing $-f_{c}(0)$, the energy of vaporisation at $T=0$, the bulk modulus, and $\alpha$ (default value $5 / 3$ ). For $\left(\rho_{0} \geq 0\right)$, Eq.(2) is replaced by

$$
f_{c}=\frac{A-2 B}{\rho_{0}} \ln \eta+\frac{A-B}{\rho_{0}}\left(\frac{1}{\eta}-1\right)+\frac{B}{\rho_{0}}(\eta-1)
$$


here $B$ is an additional parameter with the dimension of pressure. The pressure follows as the derivative (with minus sign) with respect to volume per unit of mass $v:=1 / \rho$

$$
P_{c}=\left\{\begin{array}{c}
\frac{1}{(\beta-\alpha)} A\left(\eta^{\beta}-\eta^{\alpha}\right),\left(\rho<\rho_{0}\right), \\
A(\eta-1)+B(\eta-1)^{2},\left(\rho \geq \rho_{0}\right),
\end{array}\right\}
$$

note that $P_{c}$ and it's first derivative are continuous at $\rho_{0}$. The expression for $P_{c}$ in the compression region is simply a quadratic expansion in the deviation from zero-point density, reminiscent of the 'cold' pressure term in Tillotson's EoS (see Melosh (1989) e.g.). The second parameter $B$ can be used to tune the high-pressure asymptotics of the Hugoniot curve, or it may be found from a condition of continuity of second-order derivative of $P_{c}$ at $\rho_{0}$. No attempt is made to include solid-state phase transitions, here. The authors of ANEOS (Thompson and Lauson, 1972) treat this in a rudimentary way by modification of $f_{c}$ which we could do as well if a better match of the Hugoniot curve of a given meteorite material is desired. Only the form of the potential $f_{c}(\rho)$ in expansion is important for the construction of the liquidvapor coexistence region. The contribution to the internal energy per unit mass obviously is $\epsilon_{c}=f_{c}$, because the entropy is zero at $T=0$. 


\subsection{Thermal contribution in the condensed state}

In the condensed state, the thermal contribution to the free energy is modeled in the quasi-harmonic approximation as an Einstein solid ${ }^{1}$

$$
f_{E}=-\frac{3 k_{B} T}{m} \ln (Z), \quad Z=\frac{\exp (\Theta /(2 T))}{\exp (\Theta / T)-1}
$$

where $Z$ is the partition function of a single harmonic oscillator with frequency $\nu$, and $\Theta:=h \nu / k_{B}$ is the Einstein temperature (analogous to the Debye temperature) in terms of Planck's constant $h$ and the Boltzmann constant $k_{B} ; m$ is the mean atomic mass. Equation(5) includes the contribution of the ground state. Only the lattice part of the EoS contributes to the entropy in the condensed state, thermal electronic contributions are ignored but the electronic pressure is thought to be included in the 'cold' pressure. The ratio $T / \Theta$ is constant along the adiabat as far as additional terms are suppressed in the condensed state, indeed from Eq.(5)

$$
\begin{array}{r}
s_{E}=-\left(\frac{\partial f_{E}}{\partial T}\right)_{\rho}= \\
=\frac{3 k_{B}}{m}\left[\frac{\Theta}{T} \frac{\exp (-\Theta / T)}{1-\exp (-\Theta / T)}-\ln (1-\exp (-\Theta / T))\right]
\end{array}
$$

\footnotetext{
${ }^{1} \mathrm{~A}$ Debye solid is not much different except at very low temperatures $T<<300 \mathrm{~K}$.
} 
and clearly $s_{E}$ depends on the ratio $\Theta / T$, only. For later reference, we list the lattice contribution to the thermal pressure

$$
P_{E}=\Gamma \frac{3}{2} \frac{k_{B} \Theta}{m} \rho \frac{1+\exp (-\Theta / T)}{1-\exp (-\Theta / T)}
$$

here the Debye-Grüneisen parameter $\Gamma$ is (see Landau and Lifshitz (1980), e.g.)

$$
\Gamma=\frac{\rho}{\Theta} \frac{d \Theta}{d \rho} .
$$

We employ the model of Thompson and Lauson (1972) for $\Gamma$ :

$$
\Gamma=\left\{\begin{array}{c}
1+\left(3 \Gamma_{0}-2\right)\left(\frac{\rho}{\rho_{0}}\right)+\left(1-2 \Gamma_{0}\right)\left(\frac{\rho}{\rho_{0}}\right)^{2}, \rho<\rho_{0}, \\
\Gamma_{0}\left(\frac{\rho_{0}}{\rho}\right)+\frac{2}{3}\left(1-\frac{\rho_{0}}{\rho}\right)^{2}, \rho \geq \rho_{0},
\end{array}\right\}
$$

where $\Gamma_{0}$ is the value at the density $\rho_{0}$. In compression, the leading term gives the experimentally observed $\Gamma \propto \rho^{-1}$ behaviour whereas the quadratic term is required to asymptotically reach the value $2 / 3$ of the Thomas-Fermi model. The low-density expression is simply a quadratic expansion in the density where the two coefficients are determined from continuity of $\Gamma$ and its first derivative at $\rho=\rho_{0}$. The theoretical expressions for the DebyeGrüneisen parameter and $\Theta$, see Burakovsky and Preston (2004), e.g., would lead to inconsistencies at low density (Thompson and Lauson, 1972). The 
Einstein or Debye temperature following for this model is

$$
\Theta=\left\{\begin{array}{c}
\Theta_{0}\left(\frac{\rho}{\rho_{0}}\right) \exp \left[\frac{1-2 \Gamma_{0}}{2}\left(\frac{\rho}{\rho_{0}}\right)^{2}+\left(3 \Gamma_{0}-2\right)\left(\frac{\rho}{\rho_{0}}\right)-2 \Gamma_{0}+\frac{3}{2}\right] \\
\rho<\rho_{0} \\
\Theta_{0}\left(\frac{\rho}{\rho_{0}}\right)^{2 / 3} \exp \left[\Gamma_{0}\left(1-\frac{\rho_{0}}{\rho}\right)-\frac{1}{3}\left(3-4 \frac{\rho_{0}}{\rho}+\left(\frac{\rho_{0}}{\rho}\right)^{2}\right)\right] \\
\rho \geq \rho_{0}
\end{array}\right\}
$$

and $\Theta_{0}$ is the Einstein temperature at the reference density. An indirect dependence of entropy on the 'cold curve' could enter by way of the density dependence of $\Theta$, the above model for $\Gamma$ avoids this.

\subsection{The interpolation formula}

The additional contribution to the free energy achieves the interpolation between gaseous and condensed states. It is written in the form (Thompson and Lauson, 1972)

$$
f_{\Psi}=\frac{3}{2} \frac{k_{B} T}{m} \ln (1+\Psi)
$$

where the function $\Psi$ is determined by consistency in the two limiting cases of a mixture of ideal gases and a solid. A derivation of the interpolation formula can be found in Thompson and Lauson (1972) for mixtures of monoatomic gases. Bobrovskii et al. (1974) or Melosh (2007) extended this to molecular gases in special cases. The general idea was introduced much earlier already, see (Thompson and Lauson, 1972; Bobrovskii et al., 1974, 1976) for the original references. From Eq.(5) the contribution of the Einstein 
solid in the limit $T>>\Theta$ is

$$
f_{E} \sim \frac{3 k_{B} T}{m} \ln \left(\frac{\Theta}{T}\right) .
$$

For very large temperature as compared to $\Theta$, part of the term $f_{\Psi}$ has to compensate $f_{E}$, so $\Psi$ has to contain a factor $(T / \Theta)^{2}$. Furthermore, the remaining part of the free energy has to be equal to that of an ideal gas mixture

$$
\frac{3}{2} \frac{k_{B} T}{m} \ln \Psi \rightarrow \frac{3 k_{B} T}{2 m} \ln \left(\frac{T}{\Theta}\right)^{2}+f^{i d}
$$

where $f^{i d}$ denotes the Helmholtz free energy of a mixture of ideal gases. The resulting expression for $\Psi$ is

$$
\Psi=\exp \left[\frac{2 f^{i d}}{3\left(k_{B} T / m\right)}\right]\left(\frac{T}{\Theta}\right)^{2} .
$$

In the case of mono-atomic ideal gases, the formula for $f^{i d}$ is simple. For the resulting $f^{i d}$ arising from chemical equilibrium calculations for a molecular gas, this is outlined in the appendix, in particular for thermochemical parameters in tabulated form. Thompson and Lauson (1972) and also Melosh (2007) express the last equation in terms of quantum-mechanical partition functions of gases. In the special case of mono-atomic ideal gases it can be shown that $\Psi \propto(\rho / m)^{2 / 3} \lambda^{2} T^{2} / \Theta^{2}$, where $\lambda$ denotes the thermal de Broglie wavelength of an atom (Thompson and Lauson, 1972). The authors of ANEOS treated this special case, additionally setting internal atomic par- 
tition functions equal to unity, whereas Bobrovskii et al. (1974) or Melosh (2007) insert vibrational and rotational terms for the internal partition function of molecules. We prefer the use of tabulated thermodynamic data.

The partial derivatives of the complete Helmholtz free energy $f$ with respect to $\rho$ and $T$ can be found with the help of two identities arising from the definition of $\Psi$ :

$$
\begin{gathered}
\left(\frac{\partial \Psi}{\partial T}\right)_{\rho}=\frac{2 \Psi}{3\left(k_{B} T^{2} / m\right)}\left(\frac{3 k_{B} T}{m}-\epsilon^{i d}\right) \\
\left(\frac{\partial \Psi}{\partial \rho}\right)_{T}=\frac{2 \Psi}{3\left(k_{B} T / m\right) \rho}\left(\frac{P^{i d}}{\rho}-\Gamma \frac{3 k_{B} T}{m}\right) .
\end{gathered}
$$

These identities are valid irrespective of considerations about chemical equilibrium of gases. In other words: the operations of partial derivation of $f^{i d}$ and chemical equilibrium constraint can be interchanged. This is not true for second-order derivatives of $f^{i d}$ : the partial derivative of the ideal-gas mixture pressure with respect to temperature is constrained by chemical equilibrium, likewise that of the internal energy. These derivatives are evaluated together with the chemical equilibrium calculations implemented in the EoS library. Interestingly, nowhere in the derivation it is actually needed that $f^{i d}$ describes a mixture of ideal gases, so the model for the gas phase could easily be generalized. The complete EoS with all partial derivatives necessary in hydrodynamic applications is listed in the appendix. 


\section{Hydrodynamic simulations}

The numerical hydrodynamics algorithm is a modification of our axisymmetric multi-material code (de Niem et al., 2007) valid for (quasi-1D) spherical coordinates. Table 1 shows initial values of state variables resulting from two different sets of condensed-phase EoS parameters.

In the so-called planar impact approximation (Melosh, 1989), assuming the same material for impactor and target, a particle velocity of half the impact speed results. This follows because then the shock speed $D_{s}$ is identic in both materials, and the pressure $P_{s}$ is given by $P_{s}-P_{0}=\rho D_{s}\left(u_{i}-u_{p}\right)=$ $\rho D_{s} u_{p}$ (Zel'dovich and Raizer, 1967), where $\rho$ and $P_{0}$ denote the initial density and pressure; $u_{i}$ and $u_{p}$ are the impact and particle velocities, respectively. The initial state of the vapor plume is situated on the Hugoniot curve originating at the pre-impact state (Zel'dovich and Raizer, 1967; Melosh, 1989) which we assume at a pressure of $P_{0}=1 \mathrm{~atm}$, here. Real asteroids of moderate size are porous and may resemble a rubble-pile (Britt et al., 2002). Therefore a typical porosity has been taken into account which leads to a

modified initial state of the impactor, in particular the entropy and internal energy are higher for the same value of impact velocity whereas the pressure is lower, as compared to the non-porous case, see Zel'dovich and Raizer (1967).

Two different types of impactors are considered, each corresponding to a distinct set of parameters for the condensed-phase part of the EoS. Param- 
eters for the gas-phase part are the same in both cases. In hydrodynamic calculations, the effect of chemistry on the condensed phase is ignored, not so, in the post-processing step (the chemical equilibrium calculations for liquid species). For the first type of impactor, condensed-state EoS parameters have been derived from Bobrovskii et al. (1974). This set of parameters is listed as 'granite' case in Table 2. The remaining parameter $B$ is found, imposing the additional condition of continuity of the second derivative of the pressure at $\rho_{0}$. The bulk properties are similiar ${ }^{2}$ to that of the granitic crust below the $2-3 \mathrm{~km}$ thick carbonate platform in the case of the Chicxulub impact. Recent work (Bottke et al., 2007) suggests a CM2-type carbonaceous chondrite for the impactor. Therefore the second type of impactor, denoted as the 'dunite' case in Table 2, is a more dense object where density, bulk modulus, and other parameters take values more appropriate for olivine. Values of the parameters are derived from different sources: so the value of the bulk modulus $A$ is from Anderson (1989), the density $\rho_{0}$ is typical for olivine. Parameters $\Theta_{0}$ and $\Gamma_{0}$ are the ANEOS values for dunite from Pierazzo et al. (1997, 1998) where these parameters have the same meaning as in our EoS model. The initial geometry of the vapour plume is that of a half-sphere, with radius corresponding to conservation of mass for a spherical impactor with initial diameter of $10 \mathrm{~km}$, surrounded by a constant-density atmosphere. The atmosphere is taken as an ideal gas with $\gamma=1.4$, treated as a distinct material. As mentioned, the gas-phase part of the EoS contains a mixture of

\footnotetext{
${ }^{2}$ however the gas phase composition is chondritic
} 
mono-atomic and molecular species, details concerning the set of molecular gas species are given in the section on chemical equlibria. The overall composition is taken as chondritic with abundances from Lodders (2003) with some modification for $O$ and $H$ to resemble a carbonaceous chondrite, see Table 3 below.

Results of example hydrodynamic runs are shown in Figs. 1,2, corresponding to the initial values listed in Table 1, and EoS parameters appropriate for the different types of impactors, see Table 2. The volume-of-fluid method keeps the impact-generated vapor products and atmosphere always separate, see Figs. 1,2 where we also display the volume fraction, which equals zero in a computational cell containing atmospheric gas, only. The advantage over a more simple one-material algorithm is that otherwise one would have to take the same EoS for the atmosphere and impactor, the location of the contact discontinuity to the atmosphere could barely be determined, and the distribution of mass of impact-derived vapor over the grid would remain unclear. More realistic conditions near the shock to the atmosphere are obtained in this way. The reason for the rather large but still realistic value of the impact velocity of $22 \mathrm{~km} \mathrm{~s}^{-1}$ used here is that for a lower value, condensation would begin even closer to the critical point, at conditions for density and pressure where the ideal gas approximation made in the chemical equilibrium calculations definitely breaks down.

The most obvious feature of the solution is a spatially nearly linear velocity up to the radius where the shocked atmospheric gas begins to decelerate 
impact-derived vapor. Due to the decelerating effect of the atmosphere, the maximum of velocity decreases monotoneously with time. The distribution of density, pressure and temperature are more complicated, initially. Temperature and pressure develop a local maximum near the shock front. After the highly compressed material has expanded for a while, the region interior to the contact discontinuity to the atmosphere (C.D.), where the volume fraction jumps from one to zero, takes a more flat temperature- and pressure profile. An exception from this behaviour occurs near the shock front: here impact-derived material piles up at late time. This phenomenon mainly appears due to the approximation of constant initial density for the atmosphere which does not hold in the actual geometry where the atmosphere is vertically stratified. The impact-produced vapor reaches the largest temperatures at the C.D., and the thermodynamic path there is different from that in the interior. This does not play a major role at the time where most of the mass enters the coexistence region, i.e. at the times of the two last curves shown in Figs. 1,2. There are barely differences in the two simulation cases although the initial pressure differs (cf. Table 1) by about $100 \mathrm{GPa}$. The speed of expansion is almost the same as it is found by comparing the postion of the C.D. and shock front. The value of the entropy of shocked material is comparable in both cases. Similiar thermodynamic conditions should be reached at the onset of condensation.

The main purpose of the hydrodynamic runs is to obtain the thermodynamic conditions during condensation. In Figs. 3,4 we display the ther- 
modynamic history of computational cells in $(T, \rho)$ space, for the 'granite' and 'dunite' runs, respectively. Vapor is supersaturated in the region above the coexistence curve. There are two ways to obtain the coexistence curve: a Maxwell construction using the EoS, or from the (dis)appearance of the condensed phase in the chemical equlibrium solver. Ideally, both methods should give the same curve. However differences exist despite the gas phase species are the same in both cases. Firstly, the condensed phase in the chemical equilibrium solver is a liquid solution containing many species, see next section, so the thermal part of the free energy is more realistic. Secondly, a 'cold' pressure is absent in the chemical equilibrium calculations assuming ideality of the gas phase. The Maxwell construction is a standard procedure which seeks a solution for the densities of two phases which simultaneously satisfy equality of pressure and chemical potential $\mu=f+P / \rho$ at given temperature (Landau and Lifshitz, 1980). At the phase boundary - at points where the mole fraction of condensed species is zero - only a single liquid (or solid) phase can coexist with the gas. This is a special case in more general multi-phase chemical equilibria treated in the next section.

Each symbol in Figs. 3,4 entering the supersaturated region represents a computational cell where condensation starts. In the 'granite' run, after $\sim 2.8$ seconds, the first few points approach the coexistence curve from below and the temperature is $\sim 5000 \mathrm{~K}$. Later, more and more cells move into the supersaturated vapor region. Finally, after a time of $\sim 3.2$ seconds, nearly all material is inside the mixed-phase region, the minimum temperature of 
points close to the coexistence curve is $\sim 4450 \mathrm{~K}$. Fig. 4 shows the situation in the 'dunite' case. Here condensation starts after 3.8 seconds with a maximum temperature at the coexistence curve of about $4800 \mathrm{~K}$. The interval of densities and temperatures occupied by the grid cells is comparable to the first case. After a time of about 4.2 seconds, most of the material has moved into the supersaturated region where now the lowest temperature at the coexistence curve is about $4350 \mathrm{~K}$. There is a small number of data points at each time step that escapes towards very high temperatures. These correspond to cells close to the C.D. because only here such extremes can be reached. The lower temperatures in the 'dunite' case seem to be caused in part by the slower expansion time scale for this case, although it is clear that there is no unique value where the thermodynamic path intersects the coexistence curve as it would be in an isentropic situation. The general result in both cases is that condensation occurs under extreme thermodynamic conditions, temperatures above $4000 \mathrm{~K}$, and it takes not much time, and the radius of the vapor plume when most of the material is super-saturated is about $38 \mathrm{~km}$ and $49 \mathrm{~km}$ in the 'granite' and 'dunite' cases, respectively, see Figs. 1,2 . 


\section{Chemical equilibrium and condensate com- positions}

We use chemical equilibrium calculations as a post-processing step where the coexistence curve of the vapor with liquid or other condensed phases is intersected in a given grid cell. The hydrodynamic simulation is continued, treating the material in a cell containing condensate with the EoS of the metastable vapor. This approximation is not too strong as long as the degree of condensation -the mass fraction of the condensate-- remains low. The purpose is to understand the origin of the most refractory condensates whereas it is not intended to predict the equilibrium liquid composition at low temperature ${ }^{3}$.

\subsection{Method and source of data}

We approximate the multi-component liquid that forms when the impactderived vapor starts to condense as a single ideal solution of a condensible subset of 26 elements and of 31 condensed-phase compounds. A model of the liquid phase as a single ideal solution is only the first approximation to the actual behaviour, but non-ideal solution models such as those developed by Navrotsky (1986), Ghiorso et al. (1983), Hirschmann (1991) or Sack and Ghiorso (1991) require experimental data in the temperature range of application. A better model requires knowledge of the activities of liquid

\footnotetext{
${ }^{3}$ far away from the coexistence curve
} 
components at the high temperatures involved. To first order, the liquid composition at such high temperatures and pressures as encountered in our simulations is controlled by the free enthalphy of the end-members.

The elements in the gas phase are H, C, N, O, F, Na, Mg, Al, Si, P, S, $\mathrm{Cl}, \mathrm{K}, \mathrm{Ca}, \mathrm{Sc}, \mathrm{Ti}, \mathrm{V}, \mathrm{Cr}, \mathrm{Mn}, \mathrm{Fe}, \mathrm{Ni}, \mathrm{Cu}, \mathrm{Br}, \mathrm{Sr}, \mathrm{Y}, \mathrm{Zr}$, additionally the gas contains 183 molecular compounds listed in table 1 of Sharp and Huebner (1990). The end-members of the liquid solution are a subset of the species listed in table 2 of Sharp and Huebner (1990), with the distinction that we consider the liquid state where data are available in the appropriate temperature range, whereas Sharp and Huebner (1990) solved the problem of condensation of solids from a gas of solar composition. The JANAF data (Chase, 1998) contain liquid-phase thermochemical parameters for a subset of compounds from table 2 of Sharp and Huebner (1990): CaO, FeO, FeS, MgO, TiN, VN, ZrN, NiS, TiO, VO, $\mathrm{NiS}_{2}, \mathrm{SiO}_{2}, \mathrm{TiO}_{2}, \mathrm{ZrO}_{2}, \mathrm{Al}_{2} \mathrm{O}_{3}$, $\mathrm{Cr}_{2} \mathrm{O}_{3}, \mathrm{MgSiO}_{3}, \mathrm{MgTiO}_{3}, \mathrm{Ni}_{3} \mathrm{~S}_{2}, \mathrm{Ti}_{2} \mathrm{O}_{3}, \mathrm{~V}_{2} \mathrm{O}_{3}, \mathrm{~K}_{2} \mathrm{SiO}_{3}, \mathrm{Na}_{2} \mathrm{SiO}_{3}, \mathrm{MgAl}_{2} \mathrm{O}_{4}$, $\mathrm{Mg}_{2} \mathrm{SiO}_{4}, \mathrm{~V}_{2} \mathrm{O}_{5}, \mathrm{MgTi}_{2} \mathrm{O}_{5}, \mathrm{Ti}_{3} \mathrm{O}_{5}, \mathrm{Ti}_{4} \mathrm{O}_{7}$; data for $\mathrm{MgFe}_{2} \mathrm{O}_{4}$ and $\mathrm{FeAl}_{2} \mathrm{O}_{4}$ are from Sack and Ghiorso (1991). We also include a condensible subset of the 26 elements within the liquid solution in the respective temperature range. For elements, thermochemical parameters are JANAF data, except for $C$ and $Y$, here we utilised the program COACH/THERMODATA (available online at http://thermodata.online.fr/activity.html\#logiciels). Data for some molecular gases listed in table 1 of Sharp and Huebner (1990) are absent in the 
NIST/JANAF data, in such cases we used the data of Tsuji $(1973)^{4}$ as did Sharp and Huebner (1990). In these cases where only the approximation of Tsuji (1973) is available, $\Delta H_{C}^{0}, \Delta c_{p, C}$ for molecules, with respect to atomic gases as standard state are obtained by differentiation of the respective approximating expressions for the equilibrium constant for dissociation, i.e.

$$
\frac{d \ln K_{C}}{d T}=\frac{\Delta H_{C}}{R T^{2}}, \quad \Delta c_{p, C}=\frac{d \Delta H_{C}}{d T}
$$

These quantities are required to evaluate partial derivatives of the pressure and internal energy of the ideal-gas mixture in chemical equilibrium, in the calculation of EoS derivatives in the hydrocode. For consistency of the EoS we have obtained all parameters for the molecular species from the NIST/JANAF data, where possible.

The chemical equilibrium solver is based on the SOLGASMIX algorithm which has been described in detail by Weber (1998), recently. An advantage is its robustness and simplicity for multi-phase problems. The low speed of convergence prevents its direct implementation into the hydrodynamic $\mathrm{EoS}^{5}$. Our implementation of the algorithm is a simplification to two mixture phases which may be gases, liquid or solid solutions, although generalisation to inlcude a number of stoichiometric solid phases is straightforward. The initial guess for a two-phase gas-liquid equilibrium is provided by a solution for the gas phase alone.

\footnotetext{
${ }^{4}$ and in the parametrisation of Tsuji (1973)

${ }^{5}$ where a more direct Newton method is applied
} 
The initial abundances of the vapor plume are taken from Lodders (2003, her table 3 column 6) who gave these data for primitive CI chondrites. However the hydrogen abundance is reduced to simulate a dry carbonaceous chondrite, see table 1-1 in Anderson (1989) for the water content of chondrites, e.g. The oxygen abundance is reduced by a factor of two relative to CI chondrites, consequently the abundances of the other elements increase such that a composition more typical for CM, CO, and CV carbonaceous chondrites results. The overall composition is given in table 3 for reference.

\subsection{Results}

Fig. 5 shows the elemental composition of the liquid condensate, as a function of temperature, beginning at the saturation point, corresponding to the highest temperatures shown in the diagram. The liquid mole fraction approaches zero directly at the coexistence curve, corresponding to the highest temperature, and increases with decreasing temperature; it reaches about 60 $\%$ at the lowest temperature shown in Figs. 5,6. The pressure is kept constant, a value of $\sim 50 \mathrm{~atm}$ is typical for the hydrodynamic simulations. The abundance of each element displayed in Fig. 5 is obtained as the sum over all liquid compounds containing that particular element, including pure elements, if present. Complementary, Fig. 6 displays abundances of individual compounds, except pure elements, over the same temperature range. At the highest temperature $\mathrm{Al}$ is the most abundant element besides oxygen, followed by Mg, Fe, Ca, Si. Most metals develop a local maximum abundance 
at some temperature, and later their content in the liquid is decreasing. Fe dominates over some interval of temperatures, later $\mathrm{Mg}$ crosses the curve of Fe. As Fig. 6 shows, the most abundant liquid compound species at the highest temperatures are $\mathrm{FeO}, \mathrm{MgO}, \mathrm{MgAl}_{2} \mathrm{O}_{4}, \mathrm{CaO}$, and $\mathrm{Al}_{2} \mathrm{O}_{3}$; pure metals go into solution as well. Initially few $\mathrm{MgSiO}_{3}, \mathrm{SiO}_{2}, \mathrm{Mg}_{2} \mathrm{SiO}_{4}$, and metallic Si is dissolved, and abundances of silicates increase slowly with growing liquid mole fraction. There is also some FeS, always below the fraction of $\mathrm{MgSiO}_{3}$. Sulphides are very suceptible to the amount of oxydation and would be suppressed for a less reduced primitive chondritic impactor.

\subsection{Comparison with spinel inclusions in K-P spherules}

In the following, we compare the overall elemental abundances of the liquid with that of magnesioferrite spinels contained inside spherules from the global K-P boundary layer analysed by Kyte and Bostwick (1995). The matrix material of the spherules is mostly altered and lost, and the spinels are the residues that preserve compositional information about the impact event. The spherule layer is correlated with the maximum concentration of platinum-group elements (Kyte and Bostwick, 1995). We assume that the spinels crystallised from a liquid with an overall composition quenched shortly after the onset of condensation. Condensation in impact and explosion phenomena does not occur in equilibrium, and the condensate mass fraction typically can not reach more than about 50 percent (Raizer, 1960; Zel'dovich and Raizer, 1967). Therefore we think that the overall composi- 
tion of primary condensates resembles that obtained in chemical equilibrium calculations shortly after crossing the liquid-vapor coexistence curve, when the condensate mass fraction is small. Our calculations do not indicate the presence of large amounts of silicate liquid, so the spinels could originate in a slag mixture dominated by pure metals and oxides.

Data of electron-microprobe analyses of spinel compositions have been obtained from GSA Data repository item 2005050, table DR1 (available online at www.geosociety.org/pubs/ft2005.htm). The spherules containing these spinels come from a wide range of geographic positions in the Pacific, south Atlantic, Indian ocean and Europe (Kyte and Bostwick, 1995). In Fig. 7 the composition of spinels is displayed together with that from our chemical equilibrium calculations corresponding to Fig. 5. The data scatter over a considerable range in composition for all elements except for oxygen ${ }^{6}$. To visualise the sense of compositional change with growing liquid fraction or, equivalently, decreasing temperature, the calculated values are shifted to the right in proportion to the liquid fraction. Compositions obtained in chemical equilibrium calculations for a pressure of 50 atmospheres are roughly compatible with the data from the Pacific K-P sites for such elements as $\mathrm{Fe}, \mathrm{Ni}$, Fe, Al, Cr, Ti, Mn, whereas there is great discrepancy for Ca and less for Si. The abundance of $\mathrm{Mg}$ is slightly overestimated by the model. The Ca initially contained in the metal-rich primary condensate will form separate minerals or be incorporated into silicate. A recent study by Cruz et al. (2005)

\footnotetext{
${ }^{6}$ its abundance has not been measured but is calculated assuming stoichiometric spinels
} 
for the system $\mathrm{SiO}_{2}-\mathrm{NiO}-\mathrm{FeO}$ compared Gibbs free energy of mixing of the two metal oxides in silicates with that of $\mathrm{CaO}$. For example, at $T=1873 \mathrm{~K}$ the metal oxides showed a very similiar near-ideal behavior in solution with $\mathrm{SiO}_{2}$, whereas $\mathrm{Ca}^{2+}$ incorporation into a $\mathrm{SiO}_{4}$ network is preferred, i.e. the Gibbs free energy of mixing is strongly more negative than for the metals. The data for Si given by Kyte and Bostwick (1995) probably do not characterise the spinel phase, these minor amounts may also reflect some olivine contamination. In Fig. 8 we also display a comparison of the data with calculations for the European K-P sites Caravaca and Furlo. In contrast to the marine $\mathrm{K}-\mathrm{P}$ sites the $\mathrm{Cr}$ and $\mathrm{Ni}$ abundances are underestimated by the model and the data for $\mathrm{Cr}$ are at least an order of magnitude higher than calculated.

\section{Discussion}

The EoS model combined with typical velocities for the impact of an asteroidal body leading to evaporation causes very large temperatures and pressures at the onset of condensation. This happens at least for impact velocities not much larger than $22 \mathrm{~km} \mathrm{~s}^{-1}$ and a chondritic chemical compostion of the impactor as that given in table 3. A substancially larger velocity is not typical for asteroidal impactors and the effect of shock compression in the case of the planar impact approximation already is an upper limit corresponding to a 90 degrees impact. Even considering porosity of the impactor 
the additional entropy produced in the primary shock wave does not seem to be sufficient for the condensates to appear at much lower temperatures and pressures although it lowers the impact velocity required to vaporise the impactor. It is clear that an ideal solution is a poor approximation at lower temperature where liquid immiscibility will tend to separate in particular metal oxides, silicates and pure metal. Several processes have been neglected, so the appearance of a silicate liquid at lower temperatures and the liquid immiscibility leading to separation of metal-rich phases from silicates and, most importantly, the crystallisation of spinels.

At liquid fractions not far away from the coexistence curve of the twophase mixture a range of compositions appears that corresponds - at least qualitatively - to that measured in magnesioferrite spinels by Kyte and Bostwick (1995). Therefore we suggest that the spinels originated in such a liquid. In the phase diagram of Phillips, Sõmiya, and Muan (1961), with MgO and $\mathrm{Fe}_{2} \mathrm{O}_{3}$ as end-members, a solid mixture of magnesiowüstite and magnesioferrite exists over a broad composition range. More detailled investigations of equilibrium in a cooling refractive liquid of the composition we obtained here would show that certain amount of oxydation is necessary to produce abundant spinel. This could be achieved during later reaction with atmospheric air. Krawitz and Cohen (1974) state that 'even when magnesiowüstite is rapidly cooled in a reducing atmosphere, a small volume fraction of spinel forms'.

The present model does not require a mixture with other materials such 
as target rocks of sedimentary or crustal origin. Processes of diffusion required to achieve homogeneous mixing with target material in the gas phase take much longer than the short time interval available until the impactorderived refractory material condenses. This is supported by the large Péclet number $P c=v L / D$, measuring the ratio of advective to diffusive effects; here $v$ is a typical flow velocity, $L$ the length scale (e.g. the projectile diameter) and $D$ (of the order $10^{-5} \mathrm{~m}^{2} \mathrm{~s}^{-1}$ ) the coefficient of interdiffusion of two given gas species. The hydrodynamic simulations show that there is an interval of densities, temperatures, and consequently pressures, where different parts of the vapor plume cross the coexistence curve, and there is no single thermodynamic path for the condensates. In view of the complexity of the problem of chemical composition in planetary impact the overall agreement of the model with spinel elemental composition is satisfactory.

Recently, the chondritic meteorites Gujba and Hammadah al Hamra HH237, belonging to the group of Bencubbin-like meteorites (Greshake et al., 2002; Rubin et al., 2003), have been hypothesized to result from a giant impact in the early solar system (Krot et al., 2005). Formation ages of these meteorites are $\sim 5$ Myr later than those of calcium-aluminium-rich inclusions (CAI's) (Krot et al., 2005). Gujba and HH237 are characterized by abundant Fe-Ni metal globules, Fe-Ni sulphide nodules, ferromagnesian pyroxene and olivine, and glassy or fine-grained silicate material (Rubin et al., 2003; Krot et al., 2005). As shown in this work, a metal-rich condensate containing abundant Fe-Ni, but also metal oxides and -sulphides, is the natural outcome 
in vaporising collisions. A detailled comparison of the model with the data of Rubin et al. (2003) is planned for the future.

Complete vaporisation in collisions of massive protoplanets not necessarily requires the large impact velocity of $22 \mathrm{~km} \mathrm{~s}^{-1}$ used in this work. However modeling condensation at conditions even closer to the liquid-vapour critical point is barely feasible because of the lack of knowledge of activities of liquid components at such high $P, T$. Relative velocities leading to evaporation are not characteristic for the current asteroid population (Bottke et al., 1994), but may have occurred among a lost population of mars-sized objects.

\section{Acknowledgements}

Much of this work would not have been possible without an early version of his unpublished manuscript (Melosh, 2007) which H. J. Melosh kindly provided. Comments by B. Fegley Jr., comparative chemical equilibrium calculations by L. Shaefer, and suggestions by an anonymous referee helped to improve the quality of the paper and are very much appreciated.

\section{Appendix}

\subsection{Complete EoS with partial derivatives}

In hydrodynamic applications, in addition to the pressure and internal energy, given as function of density and temperature, the partial derivatives 
of pressure and internal energy are required since most algorithms need the sound speed. Moreover, the internal energy per unit mass $\epsilon$ is given rather than the temperature, the latter has to be found numerically from $\epsilon$ and $\rho$, during the solution of the energy equation. With the help of Eqs. $(1,16)$ and the usual thermodynamic relation (Landau and Lifshitz, 1980)

$$
P=-\left(\frac{\partial f}{\partial v}\right)_{T}
$$

the total pressure $P$ is found

$$
P=P_{E}+\left(P^{i d}-3 \Gamma \frac{k_{B} T \rho}{m}\right) \frac{\Psi}{1+\Psi}+P_{c}(\rho),
$$

where $P_{E}$ is given by Eq.(7) above and $P^{i d}$ is the pressure of the ideal gas mixture of molecules in chemical equilibrium; $m:=\sum_{a} c_{a} m_{a}$ in terms of the atomic masses $m_{a}$ and fixed overall abundance of the elements, $c_{a}$. In the condensed state, at not too large a temperature, the term proportional to $\Psi$ is negligible. For large values of $\Psi$ as in the high-temperature or dilute gas limits (where $\Theta$ is negligible), the first term $P_{E}$ in Eq.(19) is compensated by $-\left(3 \Gamma k_{B} T \rho / m\right)$. The internal energy per unit mass is evaluated with the help of Eq.(15) and $\epsilon=f+T s$ where the entropy is $s=-(\partial f / \partial T)_{v}$, with the result

$$
\epsilon=\epsilon_{E}+\left(\epsilon^{i d}-\frac{3 k_{B} T}{m}\right) \frac{\Psi}{1+\Psi}+\epsilon_{c}(\rho),
$$


with $\epsilon_{E}$ following from Eqs. $(5,6)$ as

$$
\epsilon_{E}=f_{E}+T s_{E}=\frac{3}{2} \frac{k_{B} \Theta}{m} \frac{1+\exp (-\Theta / T)}{1-\exp (-\Theta / T)} .
$$

Beginning with the temperature derivative of $P$, using Eq.(19), we find

$$
\begin{gathered}
\left(\frac{\partial P}{\partial T}\right)_{\rho}=\Gamma \frac{3 k_{B} \rho}{m} \frac{\Theta^{2}}{T^{2}} \frac{\exp (-\Theta / T)}{(1-\exp (-\Theta / T))^{2}}+ \\
+\left[\left(\frac{\partial P^{i d}}{\partial T}\right)_{\rho}-\Gamma \frac{3 k_{B} \rho}{m}\right] \frac{\Psi}{(1+\Psi)}+ \\
+\left[\frac{P^{i d}}{T}-\Gamma \frac{3 k_{B} \rho}{m}\right]\left[2-\frac{2}{3} \frac{\epsilon^{i d}}{\left(k_{B} T / m\right)}\right] \frac{\Psi}{(1+\Psi)^{2}},
\end{gathered}
$$

where Eq.(15) has been used in the last term. The derivative with respect to density is found with the help of Eq.(16)

$$
\begin{array}{r}
\left(\frac{\partial P}{\partial \rho}\right)_{T}=\left(\frac{\partial P_{E}}{\partial \rho}\right)_{T}+\frac{d P_{c}}{d \rho}+ \\
+\left[\left(\frac{\partial P^{i d}}{\partial \rho}\right)_{T}-\left(\Gamma+\rho \frac{d \Gamma}{d \rho}\right) \frac{3 k_{B} T}{m}\right]_{\frac{\Psi}{(1+\Psi)}+} \\
+\frac{2}{3\left(k_{B} T / m\right) \rho^{2}}\left[P^{i d}-\Gamma \frac{3 k_{B} T \rho}{m}\right]^{2} \frac{\Psi}{(1+\Psi)^{2}},
\end{array}
$$

here the part due to the Einstein solid is

$$
\left(\frac{\partial P_{E}}{\partial \rho}\right)_{T}=\left(\Gamma+\rho \frac{d \Gamma}{d \rho}\right) \epsilon_{E}+\Gamma^{2}\left(\epsilon_{E}-c_{v, E} T\right)
$$


The specific heat per unit mass amounts to

$$
\begin{gathered}
c_{v}=c_{v, E}+\left[\left(\frac{\partial \epsilon^{i d}}{\partial T}\right)_{\rho}-3 \frac{k_{B}}{m}\right] \frac{\Psi}{(1+\Psi)}+ \\
-\frac{2}{3} \frac{1}{\left(k_{B} T^{2}\right) / m}\left[\epsilon^{i d}-3 \frac{k_{B} T}{m}\right]^{2} \frac{\Psi}{(1+\Psi)^{2}},
\end{gathered}
$$

here the contribution of the Einstein solid to the specific heat is

$$
c_{v, E}:=\frac{3 k_{B}}{m} \frac{\Theta^{2}}{T^{2}} \frac{\exp (-\Theta / T)}{(1-\exp (-\Theta / T))^{2}} .
$$

Clearly, the third terms in the expressions for the derivatives of pressure and internal energy vanish in the dilute gas limit $\Psi \rightarrow \infty$ where $\Psi /(1+\Psi)^{2} \rightarrow 0$. There is a negative part in the second term of the formula for the specific heat which does not vanish but annihilates the first term (in the Dulong-Petit limit when $\Theta / T \rightarrow 0)$.

\subsection{Evaluation of $\Psi$ from tabulated data}

It is necessary to evaluate the function $\Psi$ from tabulated thermochemical parameters. These are given for atomic and molecular species as those of an ideal gas at the standard state, leading to a molar Helmholtz free energy

$$
\begin{aligned}
F^{i d} & =\sum_{a} N_{a}\left\{\left[H_{a}^{0}-T S_{a}^{0}+R T \ln \left(\frac{R T N_{a}}{V P_{r e f}}\right)\right]-R T\right\}+ \\
& +\sum_{C} N_{C}\left\{\left[H_{C}^{0}-T S_{C}^{0}+R T \ln \left(\frac{R T N_{C}}{V P_{r e f}}\right)\right]-R T\right\},
\end{aligned}
$$


where $H_{a}^{0}, S_{a}^{0}$ refer to one mole and depend on $T$ only, $P_{\text {ref }}$ is a reference pressure (usually one atmosphere), and $R$ denotes the universal gas constant $R=N_{A} k_{B}$ in terms of Avogadro's number $N_{A}$. Here $N_{a}, N_{C}$ denote numbers of moles of mono-atomic and molecular gases, respectively. The expressions in the square bracketts are the chemical potentials on a molar basis. The law of mass action and the EoS of an ideal gas can be used to show that in chemical equilibrium

$$
F^{i d}=\sum_{a} N_{a}^{0}\left[H_{a}^{0}-T S_{a}^{0}+R T \ln \left(\frac{P_{a}}{P_{r e f}}\right)\right]-P^{i d} V
$$

where $P_{a}$ is the partial pressure of element $a$ obtained as solution of chemical equilibrium. Using Eq.(14), the function $\Psi$ can be expressed as

$$
\Psi=\left(\frac{T}{\Theta}\right)^{2} \exp \left\{\frac{2}{3} \sum_{a} c_{a}\left[\frac{G_{a}^{0}}{R T}+\ln \left(\frac{P_{a}}{P_{\text {ref }}}\right)\right]-\frac{2}{3} \frac{P^{i d}}{(R T / M) \rho}\right\}
$$

here $G_{a}^{0}:=H_{a}^{0}-T S_{a}^{0}$ is the molar Gibbs enthalpy of element $a$ at standard pressure.

\section{References}

Alvarez, L. W., Alvarez, W., Asaro, F., Michel, H. V., 1980. Extraterrestrial cause for the Cretaceous-Tertiary extinction. Science 208, 1095-1108 .

Anderson, D. L., 1989. Theory of the Earth. Blackwell Scientific Publications, 
Boston.

Bobrovskii, S. V., Gogolev, V. M., Zamyshlyaev, B. V., Lozhkina, V. B., 1974. Spalling rate in a solid medium subjected to a strong shock wave. Combustion, Explosion and Shock Waves (translation from Fizika Goreniya $i$ Vzryva) 10, No. 6, 799-805.

Bobrovskii, S. V., Gogolev, V. M., Zamyshlyaev, B. V., Lozhkina, V. B., 1976. Effect of thermal decomposition on the scabbing velocity for strong shock waves in solid media. J. Mining Science (translation from Soviet Mining Science) 12, No. 3, 273-279.

Borovička, J., Spurny, P., Koten, P., 2007. Atmospheric deceleration and light curves of Draconid meteors and implications for the structure of cometary dust. Astron. Astrophys. 473, 661-672.

Bottke, W. F., Vokrouhlický, D., Nesvorny, D., 2007. An asteroid breakup 170 Myr ago as the probable source of the $\mathrm{K} / \mathrm{T}$ impactor. Nature 449, 48-53.

Bottke, W. F., Nolan, M. C., Greenberg, R., Kolvoord, R. A., 1994. Velocity distribution among colliding asteroids. Icarus 107, 255-268.

Britt, D. T., Yoemans, D., Housen, K., Consolmagno, G., 2002. Asteroid density, porosity, and structure. In Asteroids III (eds. Bottke, W. F. Jr., Cellino, A., Paolicchi, P., and Binzel, R. P.) Univ. Arizona Press. 485-500. 
Burakovsky, L., Prestion, D. L., 2004. Analytic model of the Grüneisen parameter at all densities. J. Phys. Chem. Solids 65, 1581-1587.

Chase, M. W., Jr., 1998. NIST-JANAF Themochemical Tables, Fourth Edition, J. Phys. Chem. Ref. Data, Monograph 9, 1-1951. http://webbook.nist.gov

Cruz, R. A., Romero, S. A., Vargas, R.M., Hallen, L. M., 2005. Thermodynamic analysis of the $\mathrm{SiO}_{2}-\mathrm{NiO}-\mathrm{FeO}$ system. J. Non-Crystalline Solids 351, 1359-1365.

Ebel, D. S., Grossman, L., 2005. Spinel-bearing spherules condensed from the Chicxulub impact-vapor plume. Geology 33, 293-296.

Ghiorso, M. S., Carmichael, I. S. E., Rivers, M. L., Sack, R. O., 1983. The Gibbs free energy of mixing of natural silicate liquids; an expanded regular solution approximation for the calculation of magmatic extensive variables. Contrib. Mineral. Petrol. 84, 107-145.

Greshake, A., Krot, A. N., Meibom, A., Weisberg, M. K., Zolensky, M. E., Keil, K., 2002. Heavily-hydrated lithic clasts in $\mathrm{CH}$ chondrites and the related, metal-rich chondrites Queen Alexandra Range 94411 and Hammadah al Hamra 237. Meteor. Planet. Sci. 37, 281-293.

Hapke, B., 2001. Space weathering from Mercury to the astoerid belt. J. Geophys. Res. 106, 10039-10073. 
Hirschmann, M., 1991. Thermodynamics of multicomponent olivines and the solution properties of $(\mathrm{Ni}, \mathrm{Mg}, \mathrm{Fe}){ }_{2} \mathrm{SiO}_{4}$ and $(\mathrm{Ca}, \mathrm{Mg}, \mathrm{Fe}){ }_{2} \mathrm{SiO}_{4}$ olivines. Am. Mineralogist 76, 1232-1248.

Ivanov, B. A., Deutsch, A., 2002. The phase diagram of $\mathrm{CaCO}_{3}$ in relation to shock compression and decomposition. Phys. Earth Planet. Interiors 129, $131-143$.

Keller, L. P., McKay, D. S., 1993. Discovery of vapor deposits in the lunar regolith. Science 261, 1305-1307.

Krawitz, A., Cohen, J. B., 1974. Phase relations at low temperatures in the Fe-Mg-O system. J. Am. Ceram. Soc. 57, Iss.4, 186-189.

Krot, A. N., Amelin. Y., Cassen, P., Meibom, A., 2005. Young chondrules in CB chondrites from a giant impact in the early Solar System. Nature 436, 989-922.

Kyte, F. T., Bostwick, J. A., 1995. Magnesioferrite spinel in Cretaceous/Tertiary boundary sediments of the Pacific basin: Remnants of hot, early ejecta from the Chicxulub impact? Earth and Planet. Sci. Lett. $\mathbf{1 3 2}, 113-127$.

Landau, L. D., Lifshitz, E. M, 1980. Statistical Physics (3rd edition), Pergamon Press, New York.

Lodders, K., 2003. Solar system abundances and condensation temperatures of the elements. Astrophys. J. 591, 1220-1247. 
Melosh, H. J., 1989. Impact cratering: A geologic process. Oxford Univ. Press, New York, Clarendon Press, Oxford, 245 pp.

Melosh, H. J., 2007. A hydrocode equation of state for $\mathrm{SiO}_{2}$, Meteor. Planet. Sci. (to be published)

Montanari, A., Koeberl, C., 2000. Impact stratigraphy, the Italian record. Lecture Notes in Earth Sciences 93, Springer, Berlin, Heidelberg, 364 pp.

Moroz, L. V., Fisenko, L.F., Semjonova, L. F., Pieters, C. M., Korotaeva, N. N., 1996. Optical effects of regolith processes on S-asteroids as simulated by laser shots on ordinary chondrite and other mafic materials. Icarus $\mathbf{1 2 2}$, 366-382.

Navrotsky, A., 1986. Cation-distribution energetics and heats of mixing in $\mathrm{MgFe}_{2} \mathrm{O}_{4}-\mathrm{MgAl}_{2} \mathrm{O}_{4}, \mathrm{ZnFe}_{2} \mathrm{O}_{4}-\mathrm{ZnAl}_{2} \mathrm{O}_{4}$, and $\mathrm{NiAl}_{2} \mathrm{O}_{4}-\mathrm{ZnAl}_{2} \mathrm{O}_{4}$ spinels: Study by high-temperature calorimetry. Am. Mineralogist 71, 1160-1169.

de Niem, D., 2002. Multiple stages of condensation in impact-produced vapor clouds. In Catastrophic Events and Mass Extinctions: Impacts and Beyond (C. Koeberl, and K. G. MacLeod, eds.) Boulder, Col. Geol. Soc. Am. Spec. Pap. 356, 631-644.

de Niem, D., Kührt, E., Motschmann, U., 2007. A volume-of-fluid method for simulation of compressible axisymmetric multimaterial-flow. Computer Phys. Comm. 176,170-190. 
Phillips, B., Sõmiya, S, Muan, A., 1961. Melting relations of magnesium oxide-iron oxide mixtures in air. J. Am. Ceram. Soc. 44, 167-169.

Pierazzo, E., Vickery, A. M., Melosh, H. J., 1997. A reevaluation of impact melt production. Icarus $\mathbf{1 2 7}, 408-423$.

Pierazzo, E., Kring, D. A., Melosh, H. J., 1998. Hydrocode simulation of the Chicxulub impact event and the production of climatically active gases. $J$. Geophys. Res. 103, 28607-28626.

Raizer, Yu. P., 1960. Condensation of a cloud of vaporized matter expanding in a vacuum. Soviet Phys. JETP 37, 1229-1235.

Robin, E., Bonte, Ph., Froget, L., Jehanno, C., Rocchia, R., 1991. Formation of spinels in cosmic objects during atmospheric entry: a clue to the Cretaceous-Tertiary boundary event. Earth Plant. Sci. Lett. 108, 181-190.

Rubin, A. E., Kallemeyn, G. W., Wasson, J. T., Clayton, R. N., Mayeda, T. K., Grady, M., Verchovsky, A. B., Eugster, O., Lorenzetti, S., 2003. Formation of metal and silicate globules in Gujba: A new Bencubbin-like meteorite fall. Geochimica et Cosmochimica Acta 67, No. 17, 3283-3298.

Sack, R. O., Ghiorso, M. S., 1991, An internally consistent model for the thermodynamic properties of Fe-Mg-titanomagnetite-aluminate spinels. Contrib. Mineral. Petrol. 106, 474-505.

Sasaki, S., Hiroi, T., Nakamura, K., Hamabe, Y., Kurahashi, E., Yamada, M., 2000. Simulation of space weathering by nanosecond laser heating: 
dependence on mineral composition, weathering trend of asteroids and discovery of nanophase iron particles. Adv. Space Res. 29, No. 5, 783788.

Shaefer, L., Fegley, B. Jr., 2004. Application of an equilibrium vaporization model to the ablation of chondritic and achondritic meteorids. Earth, Moon Planets 95, 413-423.

Sharp, C. M., Huebner, W. F., 1990. Molecular equilibrium with condensation. Astrophys. J. Suppl. 72, 417-431.

Siret, D., Robin, E., 2003. Spinel formation in an impact plume: a thermodynamic approach. 34th Ann. Lunar and Planet. Sci. Conf. abstr. no 1865.

Smit, J., 1999. The global stratigraphy of the Cretaceous-Tertiary boundary impact ejecta. Ann. Rev. Planet. Sci. 27, 75-113.

Stuart, J. S., Binzel, R. P., 2004. Bias-corrected population, size distribution, and impact hazard for the near-Earth objects. Icarus 170, 295-311.

Thompson, S. L., Lauson, H. S., 1972. Improvements in the chart D radiationhydrodynamic code III: Revised analytic equations of state. Sandia $\mathrm{Na}$ tional Laboratory Report SC-RR-71 0714.

Tsuji, T., 1973. Molecular abundances in stellar atmospheres II. Astron. Astrophys. 21, 411-431. 
Wdowiak, T. J., Armendarez, L. P., Agresi, D. G., Wade, M. L., Wdowiak, S. Y., Claeys, P., Izett, G., 2001. Presence of an iron-rich nanophase material in the upper layer of the Cretaceous-Tertiary boundary clay. Meteor. Planet. Sci. 36, 123-133.

Weber, C. F., 1998. Convergence of the Equilibrium Code SOLGASMIX, J. Comput. Phys. 145, 655-670.

Zahnle, K., Dones, L., Levison, H. F., 1998. Cratering rates on the Galilean satellites. Icarus 136, 202-222.

Zel'dovich, Y. B., Raizer, Y. P., 1967. Physics of shock waves and hightemperature hydrodynamic phenomena. Academic Press, New York, 926 pp. 
Table 1: Initial state of highly shocked vapor-plume material. The two cases use different Eos parameters given in table 2.

\begin{tabular}{|c|c|c|c|c|c|l|}
\hline Case & $u_{i}\left[\mathrm{~km} \mathrm{~s}^{-1}\right]$ & $\rho\left[\mathrm{g} \mathrm{cm}^{-3}\right]$ & $P[\mathrm{GPa}]$ & $T[\mathrm{~K}]$ & $S[\mathrm{~J} / \mathrm{kg} \mathrm{K}]$ & porositiy \% \\
\hline 'granite' & 22 & 4.6416 & 205.32 & 51106 & 5752.1 & 50 \\
'dunite' & 22 & 4.6115 & 303.37 & 50907 & 5733.3 & 50 \\
\hline
\end{tabular}

Table 2: EoS parameters for the condensed-phase part. See text for details.

\begin{tabular}{|c|c|c|c|}
\hline Parameter & Units & 'granite' case & 'dunite' case \\
\hline$\rho_{0}$ & $\mathrm{gcm}^{-3}$ & 2.59 & 3.3 \\
$A$ & $\mathrm{GPa}$ & 36.208 & 129.0 \\
$B$ & $\mathrm{GPa}$ & 68.444 & 527.73 \\
$\alpha$ & & $5 / 3$ & $5 / 3$ \\
$\beta$ & & 3.1139 & 7.5151 \\
$\Gamma_{0}$ & & $2 / 3$ & 0.82 \\
$\Theta_{0}$ & $\mathrm{~K}$ & 600.0 & 661.5 \\
\hline
\end{tabular}

Table 3: Abundances of elements in the initial vapor plume.

\begin{tabular}{|c|c|c|c|}
\hline & atom \% & & atom \% \\
\hline $\mathrm{H}$ & 7.35133 & $\mathrm{Ca}$ & 0.67075 \\
$\mathrm{C}$ & 8.68136 & $\mathrm{Sc}$ & 0.00038 \\
$\mathrm{~N}$ & 0.62212 & $\mathrm{Ti}$ & 0.02724 \\
$\mathrm{O}$ & 42.44063 & $\mathrm{~V}$ & 0.00324 \\
$\mathrm{~F}$ & 0.00945 & $\mathrm{Cr}$ & 0.14763 \\
$\mathrm{Na}$ & 0.64690 & $\mathrm{Mn}$ & 0.10304 \\
$\mathrm{Mg}$ & 11.69090 & $\mathrm{Fe}$ & 9.70180 \\
$\mathrm{Al}$ & 0.93471 & $\mathrm{Ni}$ & 0.53729 \\
$\mathrm{Si}$ & 11.23901 & $\mathrm{Cu}$ & 0.00592 \\
$\mathrm{P}$ & 0.08803 & $\mathrm{Br}$ & 0.00127 \\
$\mathrm{~S}$ & 5.00065 & $\mathrm{Sr}$ & 0.00262 \\
$\mathrm{Cl}$ & 0.05885 & $\mathrm{Y}$ & 0.00005 \\
$\mathrm{~K}$ & 0.04018 & $\mathrm{Zr}$ & 0.00013 \\
\hline
\end{tabular}

\section{List of Figures}

1 Example hydrodynamic simulation at different times after the impact. Initial conditions corresponding to $22 \mathrm{~km} \mathrm{~s}^{-1}$ impact velocity, see first line in table 1 . EoS parameters for the 'granite' case, see table 2. Upper left: velocity, right: volume fraction; vapor plume occupies part where value is nonzero. Lower left: pressure, right: temperature . . . . . . . . . . 47 
2 Example hydrodynamic simulation at different times after the impact. Initial conditions corresponding to $22 \mathrm{~km} \mathrm{~s}^{-1}$ impact velocity, see second line in table 1 . EoS parameters for the 'dunite' case, see table 2. Upper left: velocity, right: volume fraction; vapor plume occupies part where value is nonzero. Lower left: pressure, right: temperature . . . . . . . . . 48

3 Coexistence curve (Maxwell construction with EoS, thick line and from chemical equilibrium, dash-dotted line) and $(T, \rho)$ values (symbols) during hydrodynamic run for the 'granite' case, see tables 1,2 for initial values and EoS parameters. Coloured symbols mark different times after impact. Region above the coexistence curve corresponds to saturated vapor. . 49

4 Coexistence curve (Maxwell construction with EoS, thick line and from chemical equilibrium, dash-dotted line) and $(T, \rho)$ values (symbols) during hydrodynamic run for the 'dunite' case, see tables 1,2 for initial values and EoS parameters. Coloured symbols mark different times after impact. Region above the coexistence curve corresponds to saturated vapor. . 50

5 Abundances of elements (sum over all condensed species), atomic percent, in the liquid as function of temperature for constant pressure of 50 atmospheres. Liquid fraction grows with decreasing temperature, $0.54 \%$ to $60 \%$ from left to right. Initial composition of the gas phase from table 3. . . . . . . . 51 
6 Abundances of liquid compounds (pure elements not shown), in mole percent, as function of temperature for constant pressure of 50 atmospheres. Liquid fraction grows with decreasing temperature, $0.54 \%$ to $60 \%$ from left to right. Initial composition of the gas phase from table 3. . . . . . . . . . 52

7 Abundance of elements in K-P spinels analysed by Kyte and Bostwick (1995) as compared to condensation model results. Open black squares: data for Pacific K-P spinels DSDP576 (upper part), DSDP803D (lower part). Lines: model liquid composition for $P=50$ atm at changing liquid fraction/temperature. To indicate sense of compositional change with liquid fraction, model values displaced into the $x$-direction in proportion to liquid fraction. . . . . . . . . . . . 53

8 Abundance of elements in K-P spinels analysed by Kyte and Bostwick (1995) as compared to condensation model results. Open black squares: data for European K-P spinels, Caravaca, Spain (upper part), Furlo, Italy (lower part). Lines: model liquid composition for $P=50$ atm at changing liquid fraction/temperature. To indicate sense of compositional change with liquid fraction, model values displaced into the $x$-direction in proportion to liquid fraction. . . . . . . . . 54 

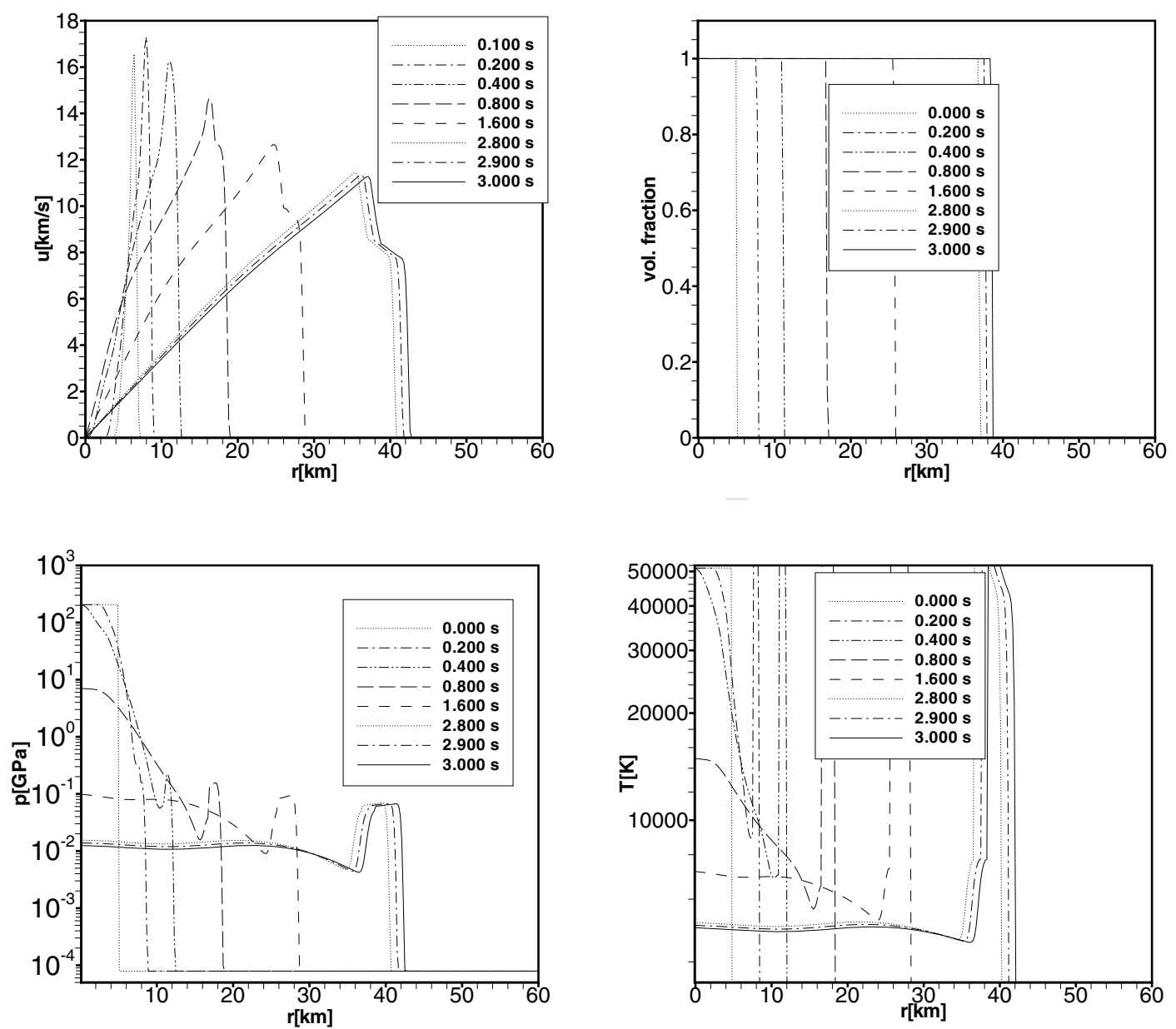

Figure 1: Example hydrodynamic simulation at different times after the impact. Initial conditions corresponding to $22 \mathrm{~km} \mathrm{~s}^{-1}$ impact velocity, see first line in table 1. EoS parameters for the 'granite' case, see table 2. Upper left: velocity, right: volume fraction; vapor plume occupies part where value is nonzero. Lower left: pressure, right: temperature 

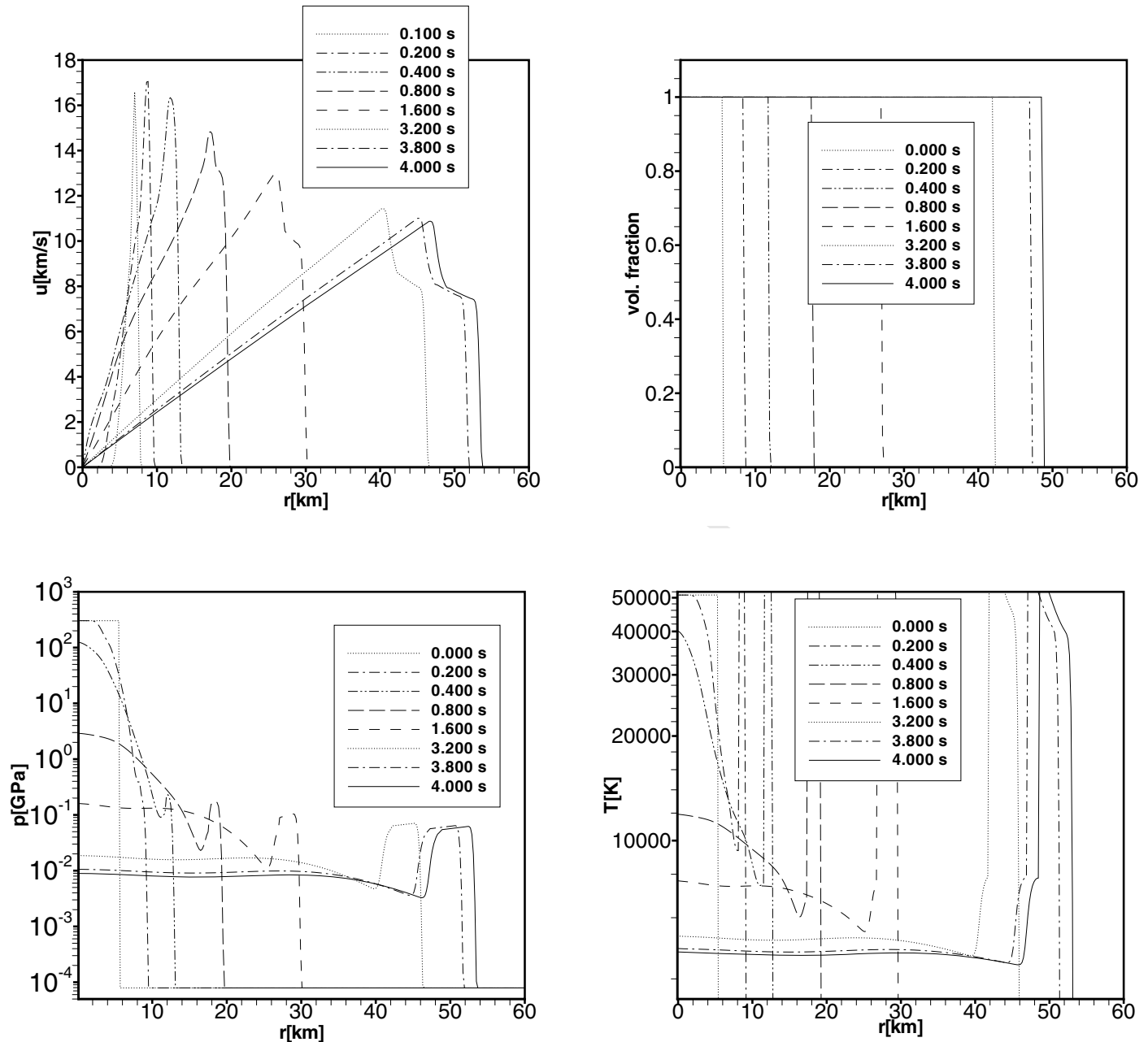

Figure 2: Example hydrodynamic simulation at different times after the impact. Initial conditions corresponding to $22 \mathrm{~km} \mathrm{~s}^{-1}$ impact velocity, see second line in table 1. EoS parameters for the 'dunite' case, see table 2. Upper left: velocity, right: volume fraction; vapor plume occupies part where value is nonzero. Lower left: pressure, right: temperature 


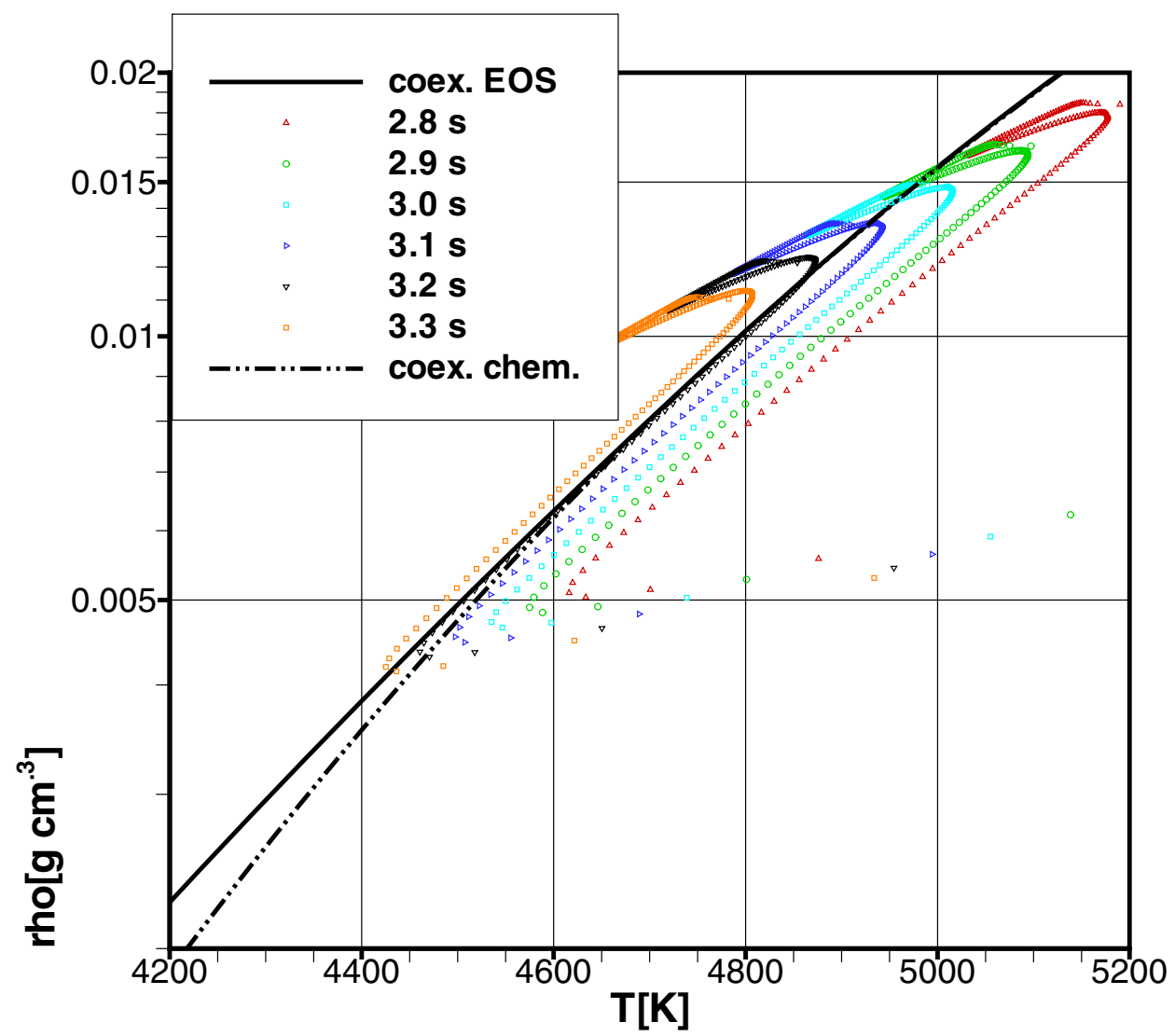

Figure 3: Coexistence curve (Maxwell construction with EoS, thick line and from chemical equilibrium, dash-dotted line) and $(T, \rho)$ values (symbols) during hydrodynamic run for the 'granite' case, see tables 1,2 for initial values and EoS parameters. Coloured symbols mark different times after impact. Region above the coexistence curve corresponds to saturated vapor. 


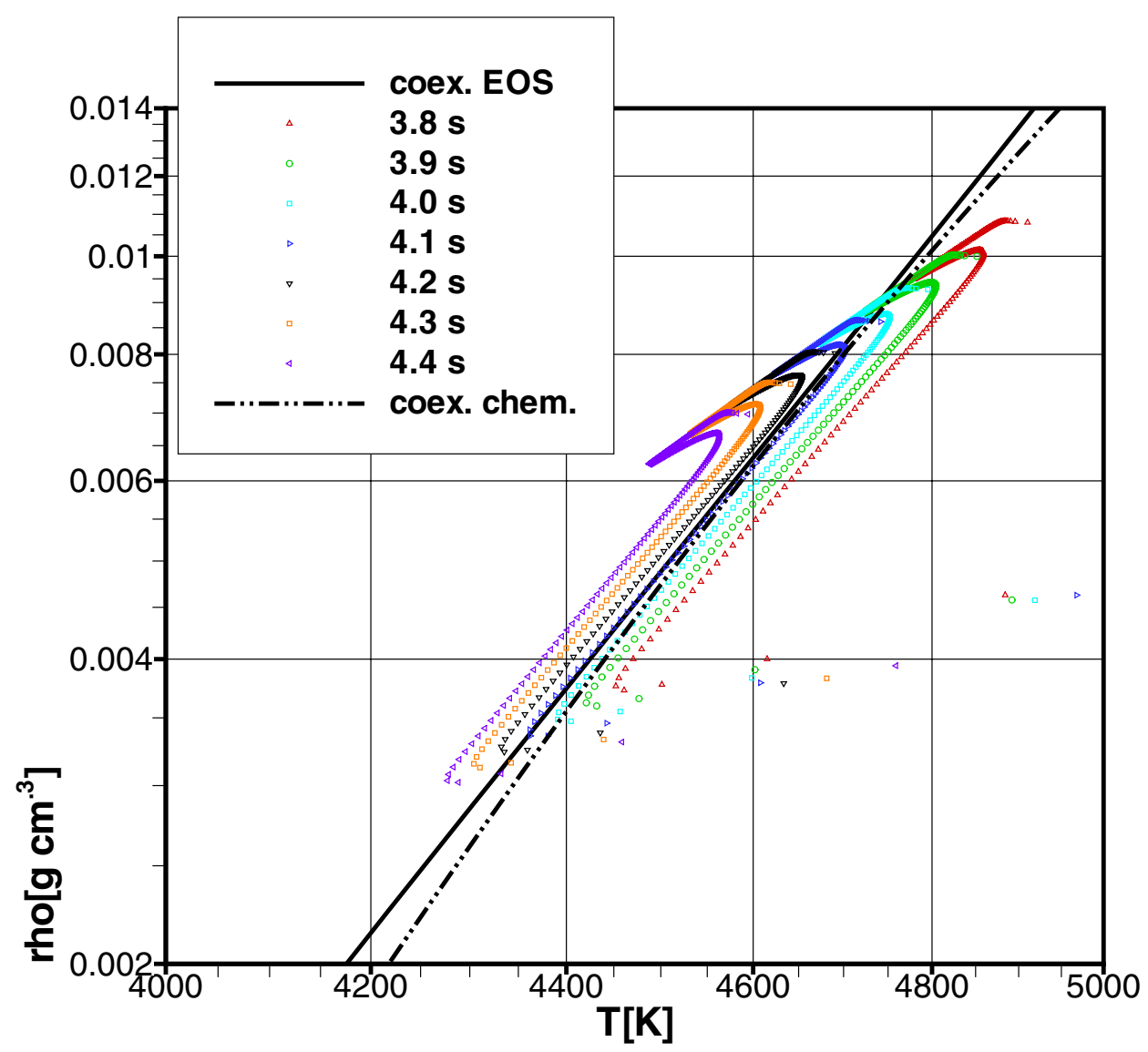

Figure 4: Coexistence curve (Maxwell construction with EoS, thick line and from chemical equilibrium, dash-dotted line) and $(T, \rho)$ values (symbols) during hydrodynamic run for the 'dunite' case, see tables 1,2 for initial values and EoS parameters. Coloured symbols mark different times after impact. Region above the coexistence curve corresponds to saturated vapor. 


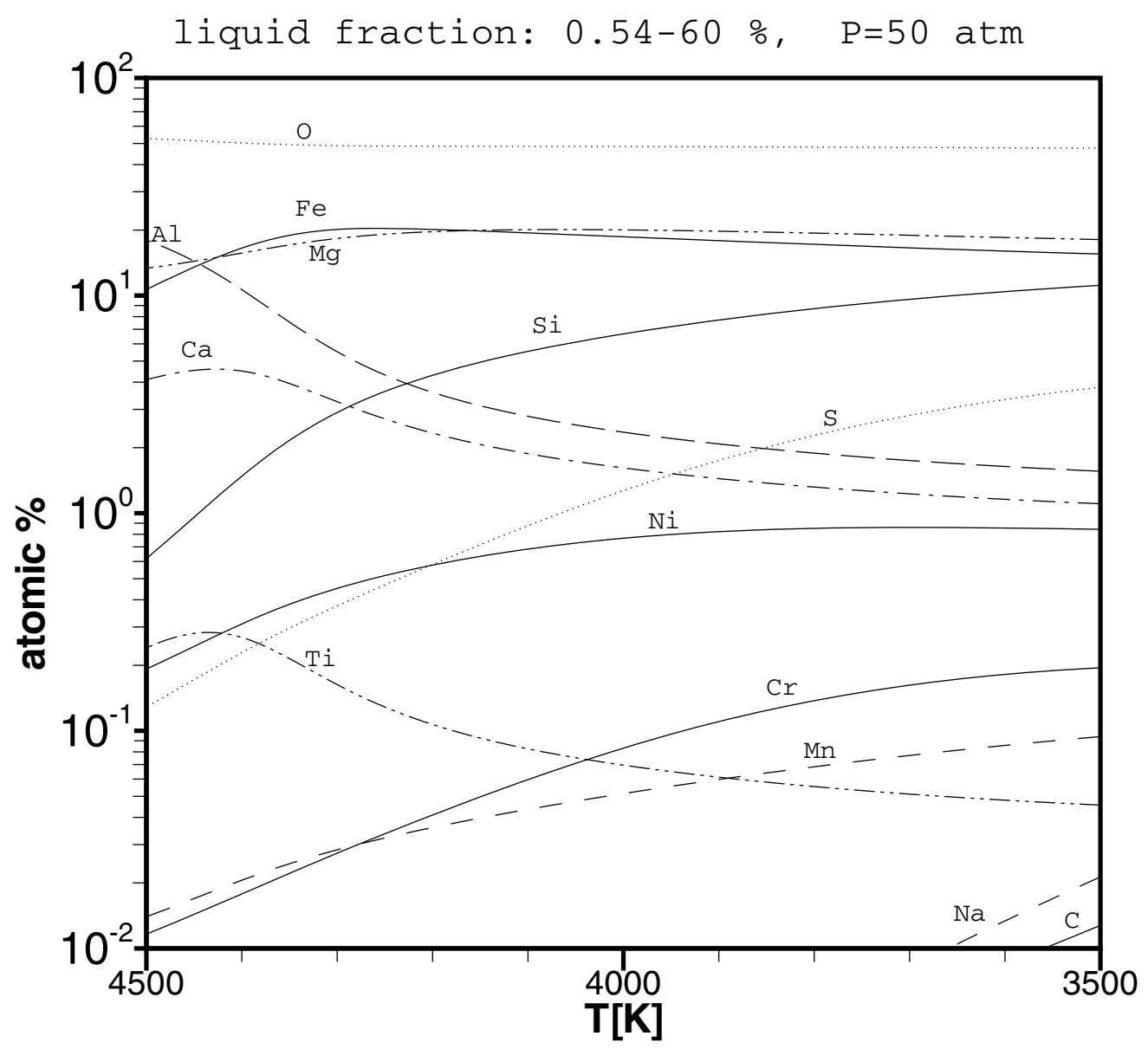

Figure 5: Abundances of elements (sum over all condensed species), atomic percent, in the liquid as function of temperature for constant pressure of 50 atmospheres. Liquid fraction grows with decreasing temperature, $0.54 \%$ to $60 \%$ from left to right. Initial composition of the gas phase from table 3 . 


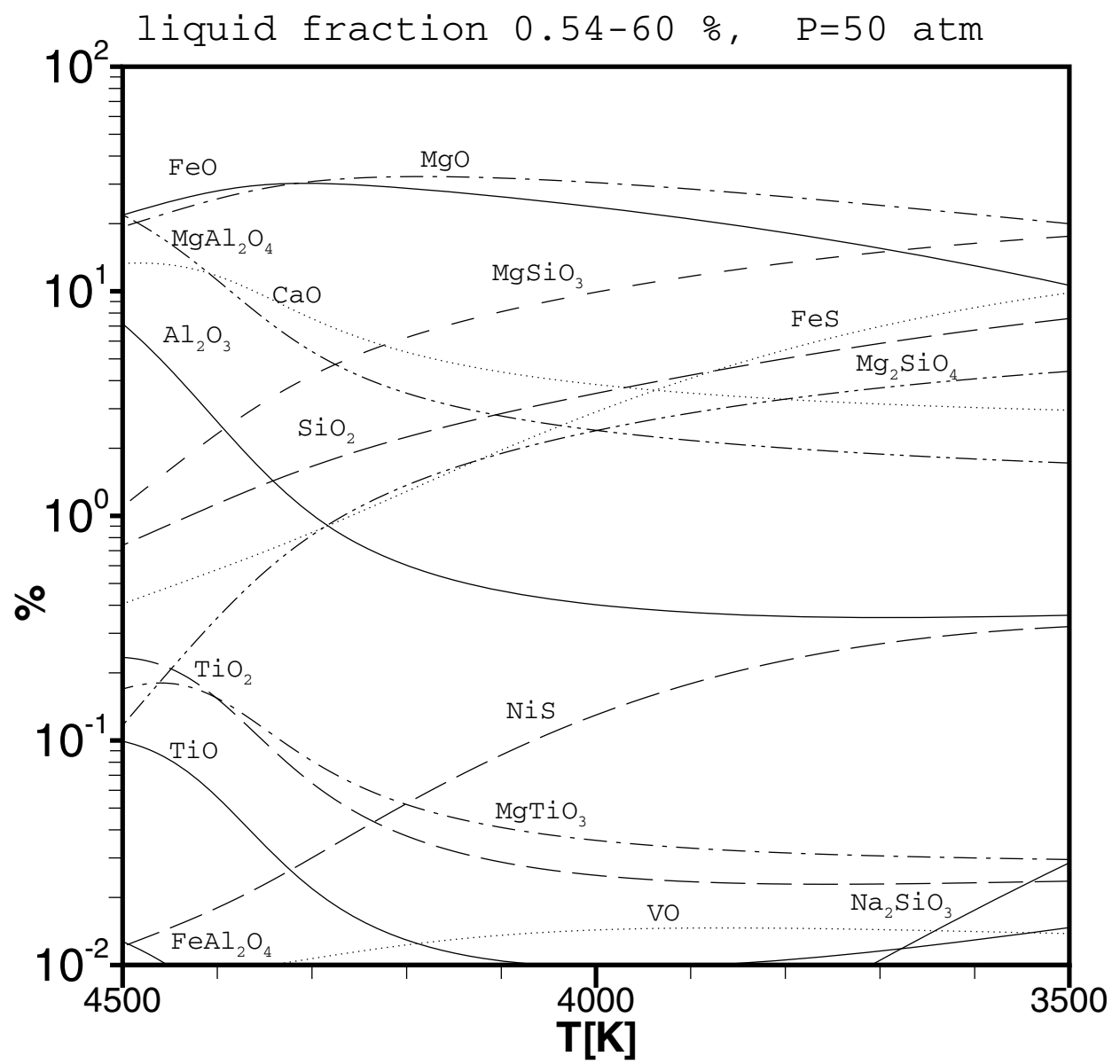

Figure 6: Abundances of liquid compounds (pure elements not shown), in mole percent, as function of temperature for constant pressure of 50 atmospheres. Liquid fraction grows with decreasing temperature, $0.54 \%$ to $60 \%$ from left to right. Initial composition of the gas phase from table 3. 

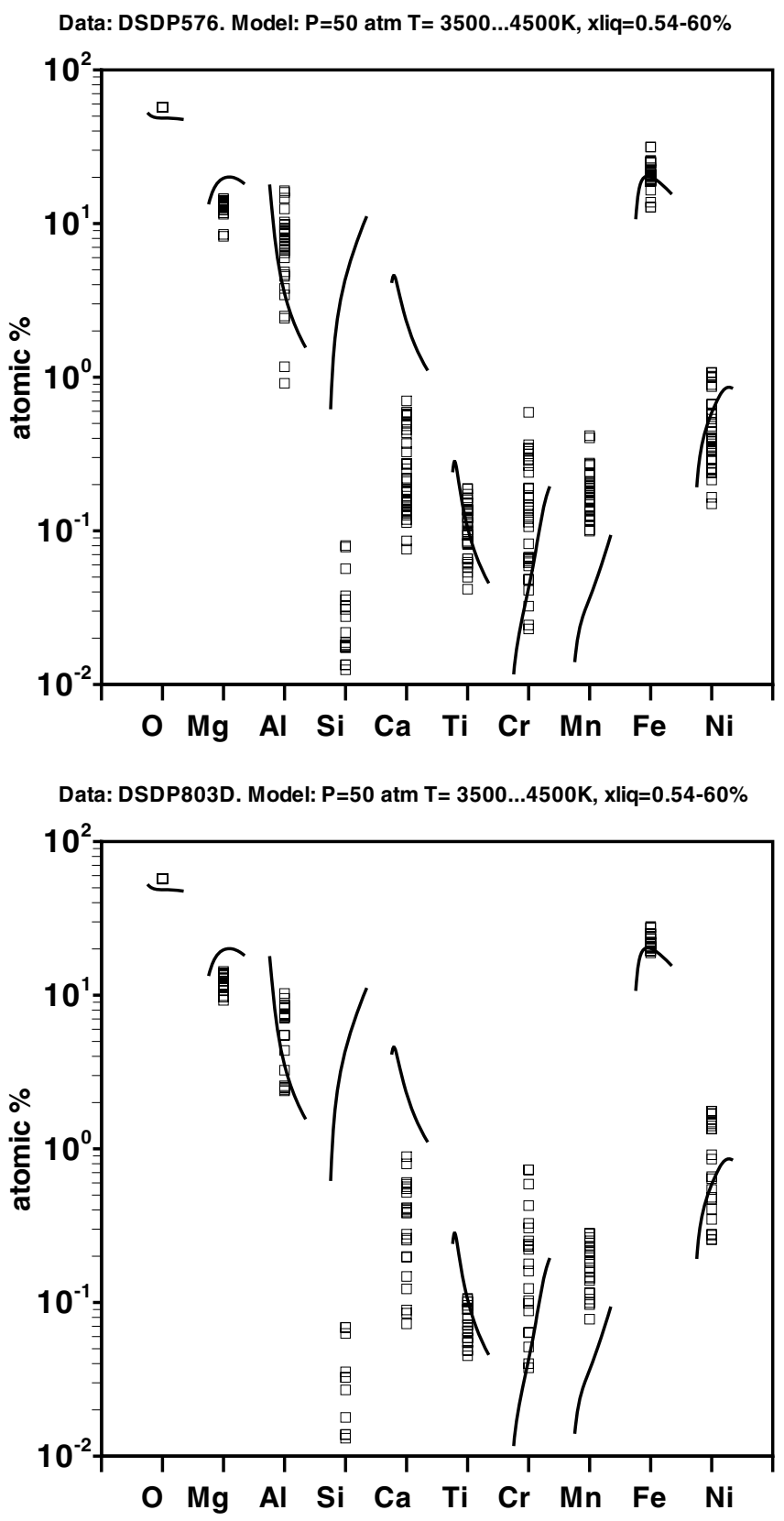

Figure 7: Abundance of elements in K-P spinels analysed by Kyte and Bostwick (1995) as compared to condensation model results. Open black squares: data for Pacific K-P spinels DSDP576 (upper part), DSDP803D (lower part). Lines: model liquid composition for $P=50$ atm at changing liquid fraction/temperature. To indicate sense of compositional change with liquid fraction, model values displaced into the $x$-direction in proportion to liquid fraction. 

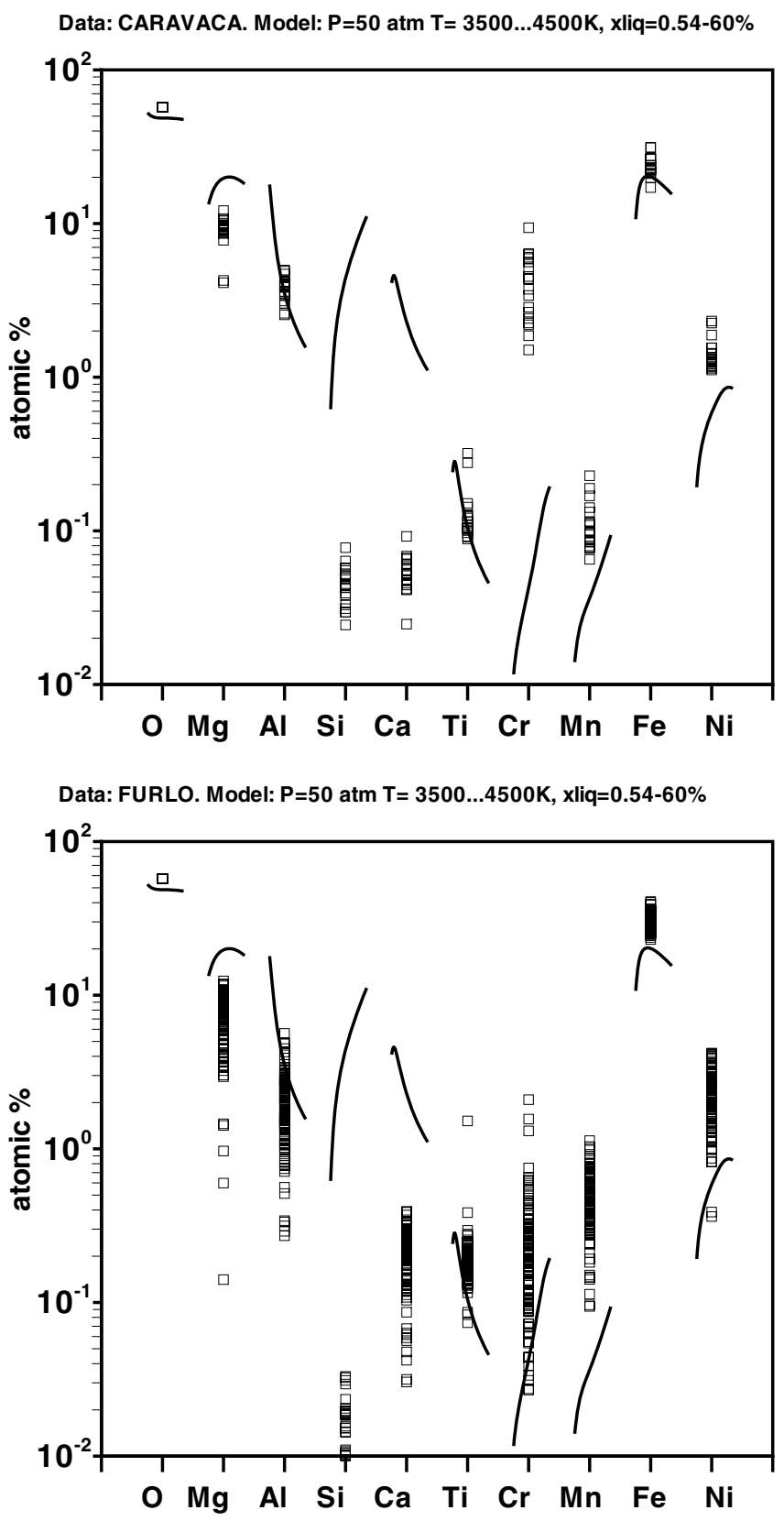

Figure 8: Abundance of elements in K-P spinels analysed by Kyte and Bostwick (1995) as compared to condensation model results. Open black squares: data for European K-P spinels, Caravaca, Spain (upper part), Furlo, Italy (lower part). Lines: model liquid composition for $P=50 \mathrm{~atm}$ at changing liquid fraction/temperature. To indicate sense of compositional change with liquid fraction, model values displaced into the $x$-direction in proportion to liquid fraction. 